\title{
On the four-dimensional formulation of dimensionally regulated amplitudes
}

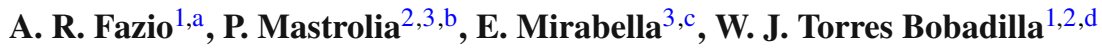 \\ ${ }^{1}$ Departamento de Física, Universidad Nacional de Colombia, Ciudad Universitaria, Bogotá, D.C., Colombia \\ 2 Dipartimento di Fisica e Astronomia, Università di Padova, and INFN, Sezione di Padova, via Marzolo 8, 35131 Padua, Italy \\ ${ }^{3}$ Max-Planck-Institut für Physik, Föhringer Ring 6, 80805 Munich, Germany
}

Received: 16 September 2014 / Accepted: 24 November 2014 / Published online: 18 December 2014

(C) The Author(s) 2014. This article is published with open access at Springerlink.com

\begin{abstract}
Elaborating on the four-dimensional helicity scheme, we propose a pure four-dimensional formulation (FDF) of the $d$-dimensional regularization of one-loop scattering amplitudes. In our formulation particles propagating inside the loop are represented by massive internal states regulating the divergences. The latter obey Feynman rules containing multiplicative selection rules which automatically account for the effects of the extra-dimensional regulating terms of the amplitude. We present explicit representations of the polarization and helicity states of the four-dimensional particles propagating in the loop. They allow for a complete, four-dimensional, unitarity-based construction of $d$-dimensional amplitudes. Generalized unitarity within the FDF does not require any higher-dimensional extension of the Clifford and the spinor algebra. Finally we show how the FDF allows for the recursive construction of $d$-dimensional one-loop integrands, generalizing the fourdimensional open-loop approach.
\end{abstract}

\section{Introduction}

The recent development of novel methods for computing one-loop scattering amplitudes has been highly stimulated by a deeper understanding of their multi-channel factorization properties in special kinematic conditions enforced by on-shellness [1-3] and generalized unitarity [4,5], strengthened by the complementary classification of the mathematical structures present in the residues at the singular points [6-11].

\footnotetext{
a e-mail: arfazio@unal.edu.co

be-mail: pierpaolo.mastrolia@cern.ch

c e-mail: mirabell@mppmu.mpg.de

de-mail: william.torres@pd.infn.it
}

The unitarity-based methods, reviewed in [12-19], use two general properties of scattering amplitudes such as analyticity and unitarity. The former grants that the amplitudes can be reconstructed from their singularity-structure while the latter grants that the residues at the singular points factorize into products of simpler amplitudes.

Integrand-reduction methods $[6,20]$, instead, allow one to decompose the integrands of scattering amplitudes are into multi-particle poles, and the multi-particle residues are expressed in terms of irreducible scalar products formed by the loop momenta and either external momenta or polarization vectors constructed out of them. The polynomial structure of the multi-particle residues is a qualitative information that turns into a quantitative algorithm for decomposing arbitrary amplitudes in terms of master integrals (MIs) by polynomial fitting at the integrand level. In this context the on-shell conditions have been used as a computational tool reducing the complexity of the algorithm. A more intimate connection among the idea of reduction under the integral sign and analyticity and unitarity has been pointed out recently. Using basic principles of algebraic geometry [7,8,21-23], have shown that the structure of the multi-particle poles is determined by the zeros of the denominators involved in the corresponding multiple cut. This new approach to integrand reduction methods allows for their systematization and for their all-loop extension.

Moreover, the proper understanding of the integrands of the amplitudes paved the way to the recent proposal of a fourdimensional renormalization scheme, which allows one the recognize and subtract UV-divergent contributions already at the integrand level [24-26].

Dimensionally regulated amplitudes are constituted by terms containing (poly)logarithms, also called cutconstructible terms, and rational terms. The former may be obtained by the discontinuity structure of integrals over the four-dimensional loop momentum. The latter ones, instead, 
escape any four-dimensional detectability and require one to cope with integrations including also the $(d-4)$ components of the loop momentum.

Within generalized-unitarity methods both terms can in principle be obtained by performing $d$-dimensional generalized cuts [27-32]. In this context, the issue of addressing factorization in conjunction with regularization clearly emerges, since $d$-dimensional unitarity requires to work with treelevel amplitudes involving external particles in arbitrary, noninteger dimensions. Polarization states, dimensionality of the on-shell momenta, and the completeness relations for the particles wavefunctions have to be consistently handled since the number of spin eigenstates depends on the space-time dimension. Therefore, in many cases generalized unitarity in arbitrary non-integer dimensions is avoided and cut-constructible and rational terms are obtained in separate steps. The former are computed by performing four-dimensional generalized cuts in the un-regularized amplitudes. If possible the rational terms are obtained by using special properties of the amplitude under consideration, like the supersymmetric decomposition $[33,34]$.

Within integrand-reduction methods, different approaches are available, according to the strategies adopted for the determination of cut-constructible and rational terms.

In some algorithms, the computation of the two ingredients proceeds in two steps [35]: the cut-constructible piece and part of the rational contributions, the so-called $R_{1}$ term, are obtained by reducing four-dimensional part of the integrand. The remaining contribution to the rational part, $R_{2}$, is instead computed by introducing new counterterm-like diagrams which depend on the model under consideration [35,36]. Alternatively, the term $R_{2}$ can be be obtained by using four-dimensional Feynman rules, as described in [37].

Other methods, instead, aim at the combined determination of the two ingredients by reducing the dimensionally regulated integrand. Therefore the numerator of the integrand has to be generated and manipulated in $d$ dimensions and acquires a dependence on $(d-4)$ and on the square of the $(d-4)$-dimensional components of the loop momentum, $\mu^{2}[31,32,38]$. The multi-particle residues are finally determined by performing generalized cuts by setting $d$-dimensional massive particles on shell. This is equivalent to have on-shell four-dimensional states whose squared mass is shifted by $\mu^{2}$.

If the integrand at a generic multiple cut is obtained as a product of tree-level amplitudes, the issues related to factorization in presence of dimensional regularization have to be addressed. An interesting approach [31] uses the linear dependence of the amplitude on the space-time dimensionality to compute the $d$-dimensional amplitude. In particular the latter is obtained by interpolating the values of the oneloop amplitude in correspondence to two different integer values of the space-time. When fermions are involved, the space-time dimensions have to admit an explicit representation of the Clifford algebra [32]. More recently, this idea has been combined with the six-dimensional helicity formalism [39] for the analytic reconstruction of one-loop scattering amplitudes in QCD via generalized unitarity.

In this article, we elaborate on the four-dimensional helicity (FDH) scheme [28,40,41] and we propose a fourdimensional formulation (FDF) of the $d$-dimensional regularization scheme which allows for a purely four-dimensional regularization of the amplitudes. Within FDF, the states in the loop are described as four-dimensional massive particles. The four-dimensional degrees of freedom of the gauge bosons are carried by massive vector bosons of mass $\mu$ and their $(d-4)$-dimensional ones by real scalar particles obeying a simple set of four-dimensional Feynman rules. A $d$-dimensional fermion of mass $m$ is instead traded for a tardyonic Dirac field with mass $m+i \mu \gamma^{5}$. The $d$ dimensional algebraic manipulations are replaced by fourdimensional ones complemented by a set of multiplicative selection rules. The latter are treated as an algebra describing internal symmetries.

Within integrand-reduction methods, our regularization scheme allows for the simultaneous computation of both the cut-constructible and the rational terms by employing a purely four-dimensional formulation of the integrands. As a consequence, an explicit four-dimensional representation of generalized states propagating around the loop can be formulated. Therefore, a straightforward implementation of $d$-dimensional generalized unitarity within exactly four space-time dimensions can be realized, avoiding any higherdimensional extension of either the Dirac [31,32] or the spinor algebra [42].

Another interesting consequence of our framework is the possibility to extend to $d$ dimensions the recursive generation of the integrand from off-shell currents and open loops, now limited to four dimensions [43-45].

The paper is organized as follows. Section 2 is devoted to the description of our regularization method, while Sect. 3 describes how generalized unitarity method can be applied in presence of a FDF of one-loop amplitudes. Sections 4, 5 and 6 , collect the applications of generalized-unitarity methods within the FDF. Section 7 describes how the integrand of the FDF of one-loop amplitudes can be generated recursively within the open-loop approach.

\section{Four-dimensional Feynman rules}

The FDH scheme $[28,40,41]$ defines a $d$-dimensional vector space embedded in a larger $d_{s}$-dimensional space, $d_{s} \equiv$ $(4-2 \epsilon)>d>4$. The scheme is determined by the following rules: 
- The loop momenta are considered to be $d$-dimensional. All observed external states are considered as four dimensional. All unobserved internal states, i.e. virtual states in loops and intermediate states in trees, are treated as $d_{s}$-dimensional.

- Since $d_{s}>d>4$, the scalar product of any $d$ - or $d_{s}$ dimensional vector with a four-dimensional vector is a four-dimensional scalar product. Moreover, any dot product between a $d_{s}$-dimensional tensor and a $d$-dimensional one is a $d$-dimensional dot product.

- The Lorentz and the Clifford algebra are performed in $d_{s}$ dimensions, which has to be kept distinct from $d$. The matrix $\gamma^{5}$ is treated using the 't Hooft-Veltman prescription, i.e. $\gamma^{5}$ commutes with the Dirac matrices carrying $-2 \epsilon$ indices.

- After the $\gamma$-matrix algebra has been performed, the limit $d_{s} \rightarrow 4$ has to be performed, keeping $d$ fixed. The limit $d \rightarrow 4$ is taken at the very end.

In the following $d_{s}$-dimensional quantities are denoted by a bar. One can split the $d_{s}$-dimensional metric tensor as follows:

$\bar{g}^{\mu \nu}=g^{\mu \nu}+\tilde{g}^{\mu \nu}$,

in terms of a four-dimensional tensor $g$ and a $-2 \epsilon$ dimensional one, $\tilde{g}$, such that

$\tilde{g}^{\mu \rho} g_{\rho v}=0, \quad \tilde{g}_{\mu}^{\mu}=-2 \epsilon \underset{d_{s} \rightarrow 4}{\longrightarrow} 0, \quad g_{\mu}^{\mu}=4$.

The tensors $g$ and $\tilde{g}$ project a $d_{S}$-dimensional vector $\bar{q}$ into the four-dimensional and the $-2 \epsilon$-dimensional subspaces, respectively,

$q^{\mu} \equiv g^{\mu}{ }_{\nu} \bar{q}^{v}, \quad \tilde{q}^{\mu} \equiv \tilde{g}^{\mu}{ }_{\nu} \bar{q}^{v}$.

At one loop the only $d$-dimensional object is the loop momentum $\bar{\ell}$. The square of its $-2 \epsilon$-dimensional component is defined as:

$\tilde{\ell}^{2}=\tilde{g}^{\mu \nu} \bar{\ell}_{\mu} \bar{\ell}_{v} \equiv-\mu^{2}$.

The properties of the matrices $\tilde{\gamma}^{\mu}=\tilde{g}^{\mu}{ }_{v} \bar{\gamma}^{v}$ can be obtained from Eq. (2)

$\left[\tilde{\gamma}^{\alpha}, \gamma^{5}\right]=0, \quad\left\{\tilde{\gamma}^{\alpha}, \gamma^{\mu}\right\}=0$,

$\left\{\tilde{\gamma}^{\alpha}, \tilde{\gamma}^{\beta}\right\}=2 \tilde{g}^{\alpha \beta}$.

We remark that the $-2 \epsilon$ tensors cannot have a fourdimensional representation. Indeed the metric tensor $\tilde{g}$ is a tripotent matrix

$\tilde{g}^{\mu \rho} \tilde{g}_{\rho \nu} \tilde{g}^{\nu \sigma}=\tilde{g}^{\mu \sigma}$,

and its square is traceless

$\tilde{g}^{\mu \rho} \tilde{g}_{\rho \mu}=\tilde{g}_{\mu}^{\mu} \underset{d_{s} \rightarrow 4}{\longrightarrow} 0$, but in any integer-dimension space the square of any non-null tripotent matrix has an integer, positive trace [46]. Moreover, the component $\tilde{\ell}$ of the loop momentum vanishes when contracted with the metric tensor $g$,

$\tilde{\ell}^{\mu} g_{\mu \nu}=\bar{\ell}_{\rho} \tilde{g}^{\rho \mu} g_{\mu \nu}=0$,

and in four dimensions the only four vector fulfilling (8) is the null one. Finally in four dimensions the only non-null matrices fulfilling the conditions (5a) are proportional to $\gamma^{5}$, hence $\tilde{\gamma} \sim \gamma^{5}$. However, the matrices $\tilde{\gamma}$ fulfill the Clifford algebra (5b), thus

$\tilde{\gamma}^{\mu} \tilde{\gamma}_{\mu} \underset{d_{s} \rightarrow 4}{\longrightarrow} 0, \quad$ while $\gamma^{5} \gamma^{5}=\mathbb{I}$.

These arguments exclude any four-dimensional representation of the $-2 \epsilon$ subspace. It is possible, however, to find such a representation by introducing additional rules, called in the following $-2 \epsilon$ selection rules, $(-2 \epsilon)$-SRs. Indeed, as shown in Appendix A, the Clifford algebra (5b) is equivalent to

$\ldots \tilde{\gamma}^{\alpha} \ldots \ldots \tilde{\gamma}_{\alpha} \ldots=0, \quad \tilde{\ell} \tilde{\ell}=-\mu^{2}$.

Therefore any regularization scheme which is equivalent of FDH has to fulfill the conditions (2)-(5a), and (10). The orthogonality conditions (2) and (3) are fulfilled by splitting a $d_{s}$-dimensional gluon onto a four-dimensional one and a colored scalar, $s_{g}$, while the other conditions are fulfilled by performing the substitutions:

$\tilde{g}^{\alpha \beta} \rightarrow G^{A B}, \quad \tilde{\ell}^{\alpha} \rightarrow i \mu Q^{A}, \quad \tilde{\gamma}^{\alpha} \rightarrow \gamma^{5} \Gamma^{A}$.

The $-2 \epsilon$-dimensional vectorial indices are thus traded for $(-2 \epsilon)$-SRs such that

$$
\begin{aligned}
& G^{A B} G^{B C}=G^{A C}, \quad G^{A A}=0, \quad G^{A B}=G^{B A}, \\
& \Gamma^{A} G^{A B}=\Gamma^{B}, \quad \Gamma^{A} \Gamma^{A}=0, \quad Q^{A} \Gamma^{A}=1, \\
& Q^{A} G^{A B}=Q^{B}, \quad Q^{A} Q^{A}=1 \text {. }
\end{aligned}
$$

The exclusion of the terms containing odd powers of $\mu$ completely defines the FDF, and it allows one to build integrands which, upon integration, yield to the same result as in the FDH scheme.

The rules (12) constitute an abstract algebra which is similar to the algebras implementing internal symmetries. For instance, in a Feynman diagrammatic approach the $(-2 \epsilon)$ SRs can be handled as the color algebra and performed for each diagram once and for all. In each diagram, the indices of the $(-2 \epsilon)$-SRs are fully contracted and the outcome of their manipulation is either 0 or \pm 1 .

To summarize, the QCD $d$-dimensional Feynman rules in the 't Hooft-Feynman gauge, collected in [47], may have the following FDF: 


$$
\begin{aligned}
& \underset{a, \alpha}{\stackrel{k}{k u_{b}}}=-i \delta^{a b} \frac{g^{\alpha \beta}}{k^{2}-\mu^{2}+i 0} \quad \text { (gluon), }
\end{aligned}
$$

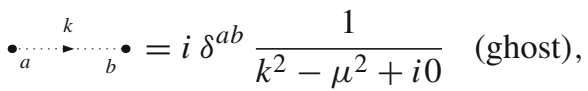

$$
\begin{aligned}
& \underset{a, A}{k} \underset{b, B}{-i}=-i \delta^{a b} \frac{G^{A B}}{k^{2}-\mu^{2}+i 0}, \quad \text { (scalar), } \\
& \stackrel{k}{\longrightarrow} \underset{j}{\stackrel{{ }_{i}}{\longrightarrow}}=i \delta^{i j} \frac{\not k+i \mu \gamma^{5}+m}{k^{2}-m^{2}-\mu^{2}+i 0}, \quad \text { (fermion), }
\end{aligned}
$$

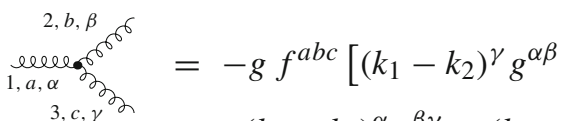

$$
\begin{aligned}
& \left.{ }^{3, c, \gamma^{\alpha}}+\left(k_{2}-k_{3}\right)^{\alpha} g^{\beta \gamma}+\left(k_{3}-k_{1}\right)^{\beta} g^{\gamma \alpha}\right] \text {, } \\
& \underset{3, c}{2, b}=-g f^{a b c} k_{2}^{\alpha} \text {, } \\
& \underset{1, a, \alpha}{2, b, B,-,}=-g f^{a b c}\left(k_{2}-k_{3}\right)^{\alpha} G^{B C} \text {, } \\
& 3, c, C \text { ' } \\
& \underset{3, a, \alpha, \gamma / 2}{2, b, B, \zeta}=\mp g f^{a b c}(i \mu) g^{\gamma \alpha} Q^{B} \\
& \left(\tilde{k}_{1}=0, \tilde{k}_{3}= \pm \tilde{\ell}\right),
\end{aligned}
$$



$$
\begin{aligned}
& +f^{x a c} f^{x b d}\left(g^{\alpha \beta} g^{\delta \gamma}-g^{\alpha \delta} g^{\beta \gamma}\right) \\
& \left.+f^{x a b} f^{x d c}\left(g^{\alpha \delta} g^{\beta \gamma}-g^{\alpha \gamma} g^{\beta \delta}\right)\right],
\end{aligned}
$$



$$
\begin{aligned}
& \underset{1, i}{2, b, \beta} \cdot 0_{3, j}^{6^{6}}=-i g\left(t^{b}\right)_{j i} \gamma^{\beta}, \\
& \underset{1, i}{2, b, B} \cdot i g\left(t^{b}\right)_{j i} \gamma^{5} \Gamma^{B} .
\end{aligned}
$$

In the Feynman rules (131) all the momenta are incoming and the scalar particle $s_{g}$ can circulate in the loop only. The terms $\mu^{2}$ appearing in the propagators (13a)-(13d) enter only if the corresponding momentum $k$ is $d$-dimensional, i.e. only if the corresponding particle circulates in the loop. In the vertex $(13 \mathrm{~h})$ the momentum $k_{1}$ is four-dimensional while the other two are $d$-dimensional. The possible combinations of the $-2 \epsilon$ components of the momenta involved are

$$
\left\{\tilde{k}_{1}, \tilde{k}_{2}, \tilde{k}_{3}\right\}=\{0, \mp \tilde{\ell}, \pm \tilde{\ell}\} \text {. }
$$

The overall sign of the Feynman rule (13h) depends on which of the combinations (14) is present in the vertex.
The $(-2 \epsilon)$-SRs (12) and the Feynman rules (131) have been implemented in FEYNARTS [48] and ForMCALC [4951] and have been used to generate the numerators of the one-loop integrands of the processes

$$
\begin{array}{ll}
q \bar{q} \rightarrow t \bar{t}, & g g \rightarrow t \bar{t}, \quad t \bar{t} \rightarrow t \bar{t}, \\
g g \rightarrow g g, & q \bar{q} \rightarrow t \bar{t} g, \quad g g \rightarrow t \bar{t} g, \\
q \bar{q} \rightarrow t \bar{t} q^{\prime} \bar{q}^{\prime} . &
\end{array}
$$

We have analytically checked that the numerators of the integrands obtained using FDF are equivalent to the corresponding ones obtained using the FDH scheme. In particular, we have verified that their difference is spurious, i.e. it vanishes upon integration over the loop momentum. As already pointed out, the $(-2 \epsilon)$-SRs constitute a formal algebra, thus they cannot have a purely numerical matrix implementation. Therefore the manipulations related to the $(-2 \epsilon)$-SRs have to be performed algebraically by using algebraic manipulations programs such as MATHEMATICA or FORM [52]. It is worth to mention that the manipulations are extremely simple and have to be performed once and for all. In particular they can be performed before any other manipulation or any recursive construction and would allow one to know in advance whether the diagram or the cut vanishes. The selection rules (12) are more trivial than the color algebra, since no interference with tree-level is needed. Moreover they can easily be implemented, e.g. along the lines of any algebraic implementation of the color manipulation.

Our prescriptions, Eq. (11), can be related to a fivedimensional theory characterized by $g^{55}=-1, \ell^{5}=$ $\mu$ and a $4 \times 4$ representation of the Clifford algebra, $\left\{\gamma^{0}, \ldots, \gamma^{3}, i \gamma^{5}\right\}$. Regularization methods in five dimensions have been proposed as an alternative formulation of the Pauli-Villars regularization [53] or as regulators of massless pure Yang-Mills theories at one loop [54]. Our method distinguishes itself by the presence of the $(-2 \epsilon)$-SRs, a crucial ingredient for the correct reconstruction of dimensionally regularized amplitudes.

It is worth to notice that the possibility to obtain the rational part of one-loop amplitudes by using four-dimensional Feynman rules has been already investigated in [37]. The method presented there computes the $\mu^{2}$-dependent part of the numerator only, thus its Feynman rules are different from the ones presented in Eq. (131). In particular our method does not introduces any additional scalar particle for each fermion flavor since the replacement of $\tilde{\gamma}^{\alpha}$ with $\gamma^{5}$ takes care of the $d_{s}$-dimensional Clifford algebra automatically. Moreover, the presence of the $(-2 \epsilon)$-SRs guarantee the proper reconstruction of the $\mu^{2}$-independent part of the numerator. Finally the propagators of the FDF, Eqs. (13a)-(13d), depend on $\mu^{2}$, thus all particles are massive. Therefore in the FDF formulation the $d$-dimensional cuts needed by both integrand reduction and generalized unitarity become four-dimensional 
massive cuts. In particular, as we show momentarily, a treelevel-based construction of the integrand has to involve amplitudes built by using $\mu$-dependent spinors and polarizations vectors, fulfilling massive completeness relations.

\section{Generalized unitarity}

Generalized-unitarity methods in $d$ dimensions require an explicit representation of the polarization vectors and the spinors of $d$-dimensional particles. The latter ones are essential ingredients for the construction of the tree-level amplitudes that are sewn along the generalized cuts. In this respect, the FDF scheme is suitable for a four dimensional realization of the $d$-dimensional generalized unitarity. The main advantage of the FDF is that the four-dimensional expression of the propagators of the particles in the loop admits an explicit representation in terms of generalized spinors and polarization expressions, whose expression is collected below.

In the following discussion we will decompose a $d$ dimensional momentum $\bar{\ell}$ as follows:

$\bar{\ell}=\ell+\tilde{\ell}, \quad \bar{\ell}^{2}=\ell^{2}-\mu^{2}=m^{2}$,

while its four-dimensional component $\ell$ will be expressed as

$\ell=\ell^{b}+\hat{q}_{\ell}, \quad \hat{q}_{\ell} \equiv \frac{m^{2}+\mu^{2}}{2 \ell \cdot q_{\ell}} q_{\ell}$,

in terms of the two massless momenta $\ell^{b}$ and $q_{\ell}$.

Spinors The spinors of a $d$-dimensional fermion have to fulfill a completeness relation which reconstructs the numerator of the cut propagator,

$$
\begin{aligned}
& 2^{\left(d_{s}-2\right) / 2} \\
& \sum_{\lambda=1} u_{\lambda,(d)}(\bar{\ell}) \bar{u}_{\lambda,(d)}(\bar{\ell})=\bar{\ell}+m, \\
& 2^{\left(d_{s}-2\right) / 2} \\
& \sum_{\lambda=1} v_{\lambda,(d)}(\bar{\ell}) \bar{v}_{\lambda,(d)}(\bar{\ell})=\bar{\ell}-m .
\end{aligned}
$$

The substitutions (11) allow one to express Eq. (18) as follows:

$$
\begin{aligned}
& \sum_{\lambda= \pm} u_{\lambda}(\ell) \bar{u}_{\lambda}(\ell)=\ell+i \mu \gamma^{5}+m \\
& \sum_{\lambda= \pm} v_{\lambda}(\ell) \bar{v}_{\lambda}(\ell)=\ell+i \mu \gamma^{5}-m .
\end{aligned}
$$

As shown in Appendix B, the generalized massive spinors

$$
\begin{aligned}
& \left.u_{+}(\ell)=\left|\ell^{b}\right\rangle+\frac{(m-i \mu)}{\left[\ell^{b} q_{\ell}\right]} \mid q_{\ell}\right], \\
& \left.u_{-}(\ell)=\mid \ell^{b}\right]+\frac{(m+i \mu)}{\left\langle\ell^{b} q_{\ell}\right\rangle}\left|q_{\ell}\right\rangle,
\end{aligned}
$$

$$
\begin{aligned}
& \left.v_{-}(\ell)=\left|\ell^{b}\right\rangle-\frac{(m-i \mu)}{\left[\ell^{b} q_{\ell}\right]} \mid q_{\ell}\right], \\
& \left.v_{+}(\ell)=\mid \ell^{b}\right]-\frac{(m+i \mu)}{\left\langle\ell^{b} q_{\ell}\right\rangle}\left|q_{\ell}\right\rangle, \\
& \bar{u}_{+}(\ell)=\left[\ell^{b} \mid+\frac{(m+i \mu)}{\left\langle q_{\ell} \ell^{b}\right\rangle}\left\langle q_{\ell}\right|,\right. \\
& \bar{u}_{-}(\ell)=\left\langle\ell^{b}\right|+\frac{(m-i \mu)}{\left[q_{\ell} \ell^{b}\right]}\left[q_{\ell} \mid,\right. \\
& \bar{v}_{-}(\ell)=\left[\ell^{b} \mid-\frac{(m+i \mu)}{\left\langle q_{\ell} \ell^{b}\right\rangle}\left\langle q_{\ell}\right|,\right. \\
& \bar{v}_{+}(\ell)=\left\langle\ell^{b}\right|-\frac{(m-i \mu)}{\left[q_{\ell} \ell^{b}\right]}\left[q_{\ell} \mid\right.
\end{aligned}
$$

fulfill the completeness relation (19). The spinors (20a) are solutions of the tardyonic Dirac equations [53,55-57]

$$
\left(\ell+i \mu \gamma^{5}-m\right) u_{\lambda}(\ell)=0, \quad\left(\ell+i \mu \gamma^{5}+m\right) v_{\lambda}(\ell)=0,
$$

which leads to a Hermitian Hamiltonian. It is worth to notice that the spinors (20) fulfill the Gordon identities

$$
\frac{\bar{u}_{\lambda}(\ell) \gamma^{\nu} u_{\lambda}(\ell)}{2}=\frac{\bar{v}_{\lambda}(\ell) \gamma^{\nu} v_{\lambda}(\ell)}{2}=\ell^{\nu} .
$$

Polarization vectors The $d$-dimensional polarization vectors of a spin-1 particle fulfill the following relation:

$$
\sum_{i=1}^{d-2} \varepsilon_{i(d)}^{\mu}(\bar{\ell}, \bar{\eta}) \varepsilon_{i(d)}^{* v}(\bar{\ell}, \bar{\eta})=-\bar{g}^{\mu \nu}+\frac{\bar{\ell}^{\mu} \bar{\eta}^{\nu}+\bar{\ell}^{\nu} \bar{\eta}^{\mu}}{\bar{\ell} \cdot \bar{\eta}}
$$

where $\bar{\eta}$ is an arbitrary $d$-dimensional massless momentum such that $\bar{\ell} \cdot \bar{\eta} \neq 0$. Gauge invariance in $d$ dimensions guarantees that the cut is independent of $\bar{\eta}$. In particular the choice

$\bar{\eta}^{\mu}=\ell^{\mu}-\tilde{\ell}^{\mu}$,

with $\ell, \tilde{\ell}$ defined in Eq. (16), allows one to disentangle the four-dimensional contribution form the $d$-dimensional one:

$$
\begin{aligned}
& \sum_{i=1}^{d-2} \varepsilon_{i(d)}^{\mu}(\bar{\ell}, \bar{\eta}) \varepsilon_{i(d)}^{* v}(\bar{\ell}, \bar{\eta}) \\
& =\left(-g^{\mu \nu}+\frac{\ell^{\mu} \ell^{v}}{\mu^{2}}\right)-\left(\tilde{g}^{\mu \nu}+\frac{\tilde{\ell}^{\mu} \tilde{\ell}^{v}}{\mu^{2}}\right) .
\end{aligned}
$$

The first term is related to the cut propagator of a massive gluon and can be expressed as follows:

$$
-g^{\mu \nu}+\frac{\ell^{\mu} \ell^{\nu}}{\mu^{2}}=\sum_{\lambda= \pm, 0} \varepsilon_{\lambda}^{\mu}(\ell) \varepsilon_{\lambda}^{* \nu}(\ell),
$$


in terms of the polarization vectors of a vector boson of mass $\mu[58]$,

$\varepsilon_{+}^{\mu}(\ell)=-\frac{\left[\ell^{b}\left|\gamma^{\mu}\right| \hat{q}_{\ell}\right\rangle}{\sqrt{2} \mu}, \quad \varepsilon_{-}^{\mu}(\ell)=-\frac{\left\langle\ell^{b}\left|\gamma^{\mu}\right| \hat{q}_{\ell}\right]}{\sqrt{2} \mu}$,

$\varepsilon_{0}^{\mu}(\ell)=\frac{\ell^{b \mu}-\hat{q}_{\ell}^{\mu}}{\mu}$.

The latter fulfill the well-known relations

$\varepsilon_{ \pm}^{2}(\ell)=0, \quad \varepsilon_{ \pm}(\ell) \cdot \varepsilon_{\mp}(\ell)=-1$,

$\varepsilon_{0}^{2}(\ell)=-1, \quad \varepsilon_{ \pm}(\ell) \cdot \varepsilon_{0}(\ell)=0$,

$\varepsilon_{\lambda}(\ell) \cdot \ell=0$.

The second term of the r.h.s. of Eq. (25) is related to the numerator of cut propagator of the scalar $s_{g}$ and can be expressed in terms of the $(-2 \epsilon)$-SRs as:

$\tilde{g}^{\mu \nu}+\frac{\tilde{\ell}^{\mu} \tilde{\ell}^{\nu}}{\mu^{2}} \rightarrow \hat{G}^{A B} \equiv G^{A B}-Q^{A} Q^{B}$.

The factor $\hat{G}^{A B}$ can easily be accounted for by defining the cut propagator as

$$
\begin{array}{c:c}
\bullet, A & b, B
\end{array}=\hat{G}^{A B} \delta^{a b} .
$$

The generalized four-dimensional spinors and polarization vectors defined above can be used for constructing treelevel amplitudes with full $\mu$-dependence.

The FDF within generalized unitarity may be seen as a massive implementation of $d$-dimensional regularization. However, the FDF is different from the most commonly used massive regularization prescriptions, i.e. the one introducing a massive scalar particle [59] and the six-dimensional helicity method [39]. Indeed the former relies on the supersymmetric decomposition of the amplitude in terms of cutconstructible supersymmetric amplitudes and an amplitude involving a scalar. The original amplitude is computed in two steps. The cut-constructible part is obtained by using fourdimensional unitarity while the rational one is computed by using the amplitude involving a $d$-dimensional scalar, which is traded with a massive four-dimensional ones. The FDF does not rely on existence of the supersymmetric decomposition and computes the full amplitude without splitting it.

The six-dimensional helicity method casts $d$-dimensional on-shell momenta into a six-dimensional massless spinor and, on the cuts, uses six-dimensional helicity spinors to compute efficiently the relevant tree-level amplitudes. However, since dimensional regularization cannot be achieved in finite dimensions, the six-dimensional helicity method deliver a result that has to be corrected by hand with the help of topologies involving six-dimensional scalars along the lines of [31]. The FDF, instead, splits the $d$-dimensional objects into their four-dimensional and $(d-4)$-dimensional parts and finds a four-dimensional representation for both of them. Moreover, it introduces the $(-2 \epsilon)$-SRs to account for the orthogonality of the subspaces and for the effects of the $\left(d_{s}-4\right) \rightarrow 0$ limit. No further corrections are needed since FDF properly takes care of the peculiar features of $d$-dimensional regularization. Therefore, in the context of on-shell and unitaritybased methods, they are a simple alternative to approaches introducing explicit higher-dimensional extension of either the Dirac $[31,32]$ or the spinor $[39,42]$ algebra.

\section{The gggg amplitude}

As a first example we consider the four-gluon color-ordered helicity amplitude $A_{4}\left(1_{g}^{+}, 2_{g}^{+}, 3_{g}^{+}, 4_{g}^{+}\right)$. The latter vanishes at tree-level, while the one-loop contribution is finite, rational and can be obtained from the quadruple cut $C_{1|2| 3 \mid 4}[28,40$, 59-61]. The relevant tree-level three-point amplitudes are computed by using the color-ordered Feynman rules collected in Appendix $\mathrm{C}$ and collected in Appendix E.

In the FDF, the quadruple-cut $C_{1|2| 3 \mid 4}$ and the coefficients $c_{1|2| 3 \mid 4 ; n}$ can be decomposed into a sum of five contributions,

$C_{1|2| 3 \mid 4}=\sum_{i=0}^{4} C_{1|2| 3 \mid 4}^{[i]}, \quad c_{1|2| 3 \mid 4 ; n}=\sum_{i=0}^{4} c_{1|2| 3 \mid 4 ; n}^{[i]}$,

where $C^{[i]}\left(c^{[i]}\right)$ is the contribution to the cut (coefficient) involving $i$ internal scalars. In the picture below, internal lines are understood to be on-shell. The quadruple cuts read as follows:




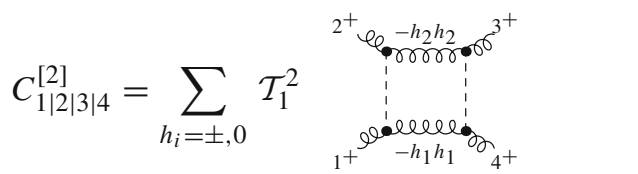

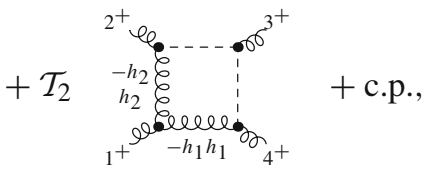


$C_{1|2| 3 \mid 4}^{[3]}=\sum_{h_{1}= \pm, 0} \mathcal{T}_{3} \vdots_{1^{+}}^{2^{+} \varphi_{h_{1} h_{1}}}+\sigma_{4^{+}}^{3^{+}}+$c.p.,

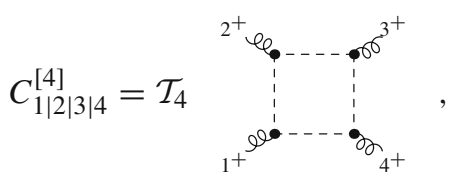

where the abbreviation "c.p." means "cyclic permutations of the external particles". In Eqs. (32) the $(-2 \epsilon)$-SR have been stripped off and collected in the prefactors $\mathcal{T}_{i}$,

$\mathcal{T}_{1}=Q^{A} \hat{G}^{A B} Q^{B}=0$,

$\mathcal{T}_{2}=Q^{A} \hat{G}^{A B} G^{B C} \hat{G}^{C D} Q^{D}=0$,

$\mathcal{T}_{3}=Q^{A} \hat{G}^{A B} G^{B C} \hat{G}^{C D} G^{D E} \hat{G}^{E F} Q^{F}=0$,

$\mathcal{T}_{4}=\operatorname{tr}(G \hat{G} G \hat{G} G \hat{G} G \hat{G})=-1$.

The prefactors $\mathcal{T}_{1}, \mathcal{T}_{2}, \mathcal{T}_{3}$ force the cuts (32b)-(32d) to vanish identically. The only cuts contributing, Eqs. (32) and (32e), lead to the following coefficients:

$c_{1|2| 3 \mid 4 ; 0}^{[0]}=0, \quad c_{1|2| 3 \mid 4 ; 4}^{[0]}=3 i \frac{[12][34]}{\langle 12\rangle\langle 34\rangle}$,

$c_{1|2| 3 \mid 4 ; 0}^{[4]}=0, \quad c_{1|2| 3 \mid 4 ; 4}^{[4]}=-i \frac{[12][34]}{\langle 12\rangle\langle 34\rangle}$.

The color-ordered one-loop amplitude can be obtained from Eqs. (31) and (D.11). In this simple case it reduces to

$$
\begin{aligned}
A_{4}\left(1_{g}^{+}, 2_{g}^{+}, 3_{g}^{+}, 4_{g}^{+}\right) & =c_{1|2| 3 \mid 4 ; 4} I_{1|2| 3 \mid 4}\left[\mu^{4}\right] \\
& =-\frac{i}{48 \pi^{2}} \frac{[12][34]}{\langle 12\rangle\langle 34\rangle}
\end{aligned}
$$

and it is in agreement with the literature [60]. This example clearly shows the difference between our computation and the one based on the supersymmetric decomposition [59]. In the latter one, the result is uniquely originating by the complex scalar contribution. Instead in our procedure the result arises from both the massive gluons and the massive scalars $s_{g}$.

For clarity reasons, in this example we have computed the $(-2 \epsilon)$-SRs factors, $\mathcal{T}_{i}$, explicitly. It is worth to notice that in practice the $(-2 \epsilon)$-SRs can easily be automated and can be performed cut-by-cut once and for all, even before the treelevel amplitudes are computed. Therefore the cut topologies which vanish because of the $(-2 \epsilon)$-SRs can be discarded at the beginning of the computation without affecting its complexity.

\section{The ggq $\overline{\mathbf{q}}$ amplitude}

In this section we show the calculation of the leadingcolor one-loop contribution to the helicity amplitude
$A_{4}\left(1_{g}^{-}, 2_{g}^{+}, 3_{\bar{q}}^{-}, 4_{q}^{+}\right)$, which at tree-level reads,

$A_{4}^{\text {tree }}=-i \frac{\langle 13\rangle^{3}\langle 14\rangle}{\langle 12\rangle\langle 23\rangle\langle 34\rangle\langle 41\rangle}$.

The leading-color contribution to a one-loop amplitude with $n$ particles and two external fermions can be decomposed in terms of primitive amplitudes [62]. For the helicity configuration we consider the amplitude can be expressed in terms of the left-turning, $A_{4}^{\mathrm{L}}$, and right-turning, $A_{4}^{\mathrm{R}}$, primitive amplitudes as follows:

$A_{4}^{1 \text { loop }}=A_{4}^{\mathrm{L}}-\frac{1}{N_{c}^{2}} A_{4}^{\mathrm{R}}$,

where $N_{c}$ is the number of colors.

Left-turning amplitude In the following we list the coefficients $c_{i_{1} \ldots i_{k} ; n}^{[\mathrm{L}]}$ entering the decomposition (D.11) of $A_{4}^{\mathrm{L}}$ and the corresponding cut $C_{i_{1} \ldots i_{k}}^{[\mathrm{L}]}$. The quadruple cut is given by



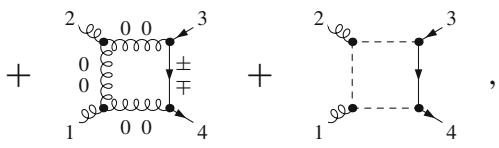

$c_{1|2| 3 \mid 4 ; 0}^{[\mathrm{L}]}=\frac{1}{2} A_{4}^{\text {tree }}\left(1-\frac{s_{14}^{3}}{s_{13}^{3}}\right) s_{12} s_{14}$,

$c_{1|2| 3 \mid 4 ; 4}^{[\mathrm{L}]}=0$.

The first two cut diagrams contribute both to the cutconstructible and to the rational part, while the last two cut diagrams cancel against each other.

The triple cuts are given by

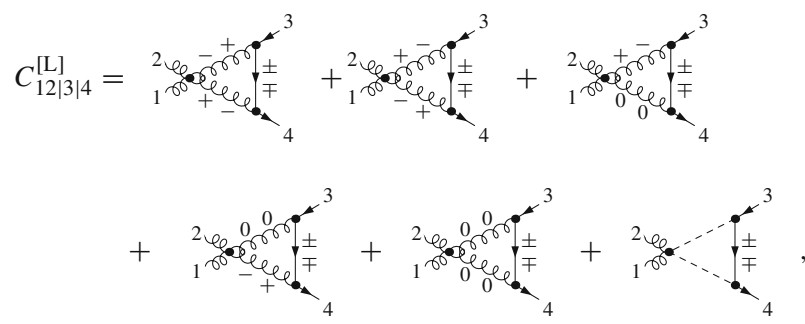

$c_{12|3| 4 ; 0}^{[\mathrm{L}]}=\frac{1}{2} A_{4}^{\text {tree }}\left(1-\frac{s_{14}^{3}}{s_{13}^{3}}\right) s_{12}$,

$c_{12|3| 4 ; 2}^{[\mathrm{L}]}=\frac{1}{2} A_{4}^{\text {tree }}\left(2-\frac{s_{12}^{2}}{s_{13}^{2}}\right)$; 


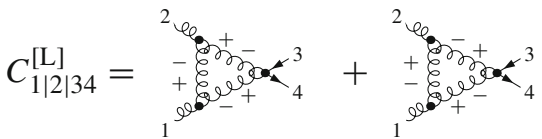

$$
\begin{aligned}
& +{ }_{1}^{2} \operatorname{cog}_{0}^{0} \operatorname{lok}_{4}^{3}+{ }_{1}^{2} \operatorname{coc}_{4}^{3}, \\
& c_{1|2| 34 ; 0}^{[\mathrm{L}]}=-\frac{1}{2} A_{4}^{\text {tree }}\left(1+\frac{s_{14^{3}}}{s_{13}{ }^{3}}\right) s_{12}, \\
& c_{1|2| 34 ; 2}^{[\mathrm{L}]}=-\frac{1}{2} A_{4}^{\text {tree }} \frac{s_{12}^{2}}{s_{13}^{2}} \text {; }
\end{aligned}
$$

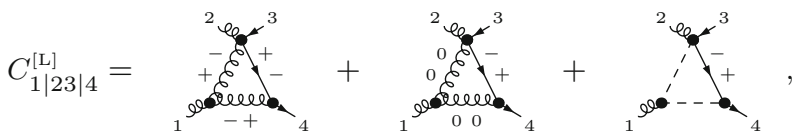

$$
\begin{aligned}
& c_{1|23| 4 ; 0}^{[\mathrm{L}]}=-\frac{1}{2} A_{4}^{\mathrm{tree}}\left(1+\frac{s_{14}^{3}}{s_{13}^{3}}\right) s_{14}, \\
& c_{1|23| 4 ; 2}^{[\mathrm{L}]}=-\frac{1}{2} A_{4}^{\text {tree }} \frac{s_{14} s_{12}}{s_{13}^{2}} \text {; }
\end{aligned}
$$

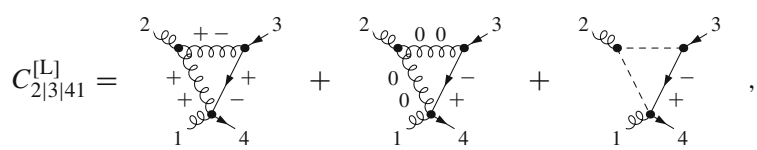$$
c_{2|3| 41 ; 0}^{[\mathrm{L}]}=-\frac{1}{2} A_{4}^{\text {tree }}\left(1+\frac{s_{14}^{3}}{s_{13}^{3}}\right) s_{14},
$$$$
c_{2|3| 41 ; 2}^{[\mathrm{L}]}=-\frac{1}{2} A_{4}^{\mathrm{tree}} \frac{s_{14} s_{12}}{s_{13}^{2}} \text {. }
$$

In all the triple cuts the last two cut diagrams cancel against each other. In the cut $C_{12|3| 4}^{[\mathrm{L}]}$, Eq. (39c), the third cut diagram exactly compensates the contribution of the fourth one.

The double cuts read as follows:





$$
\begin{aligned}
& c_{12 \mid 34 ; 0}^{[\mathrm{L}]}=A_{4}^{\text {tree }} \frac{s_{14}}{s_{13}}\left(\frac{s_{14}}{s_{13}}-\frac{1}{2}\right), \\
& c_{12 \mid 34 ; 2}^{[\mathrm{L}]}=0 \text {; }
\end{aligned}
$$

$$
\begin{aligned}
& C_{23 \mid 41}^{[\mathrm{L}]}={ }^{-}+\xi_{1}^{2} \sum_{-}^{+}+{ }_{0}^{0} \xi_{1}^{2} \xi_{-}^{2}<_{4}^{3} \\
& +\xi_{0}^{0} \xi_{+}^{2}+\sum_{1}^{3}+
\end{aligned}
$$

\section{The gggH amplitude}

In this section, we show how the FDF scheme can be applied in the context of an effective theory, where the Higgs boson couples directly to the gluon. In particular we compute leading-color one-loop contribution to the helicity amplitude $A_{4}\left(1_{g}^{-}, 2_{g}^{+}, 3_{g}^{+}, H\right)$ in the heavy top-mass limit, which at leading order is given by

$A_{4, H}^{\text {tree }}=i \frac{[23]^{4}}{[12][23][31]}$

The Feynman rules for the Higgs-gluon and Higgs-scalar couplings in the FDF are given in Appendix C. They are used to compute the tree-level amplitudes sewn along the cuts.

The one-loop amplitude can be decomposed according to Eq. (D.13), in terms of three different ordering of the external particles, i.e. $123 \mathrm{H}, 12 \mathrm{H} 3$ and $1 \mathrm{H} 23$. In the case of the first ordering the coefficients $c_{i_{1} \ldots i_{k}} ; n$ and the corresponding cut $C_{i_{1} \ldots i_{k}}$ read as follows:

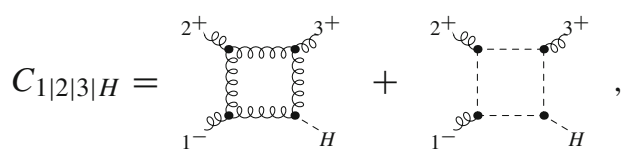

$c_{1|2| 3 \mid H ; 0}=-\frac{1}{2} A_{4, H}^{\text {tree }} s_{12} s_{23}$,

$c_{1|2| 3 \mid H ; 4}=0$; 


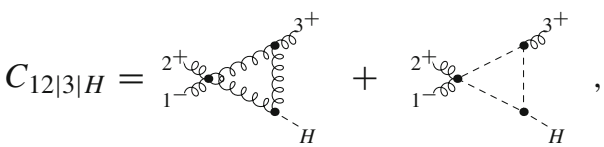

$c_{12|3| H ; 0}=\frac{1}{2} A_{4, H}^{\text {tree }}\left(s_{13}+s_{23}\right)$,

$c_{12|3| H ; 2}=0$;



$c_{1|23| H ; 0}=\frac{1}{2} A_{4, H}^{\text {tree }}\left(s_{12}+s_{13}\right)$,

$c_{1|23| H ; 2}=0$;

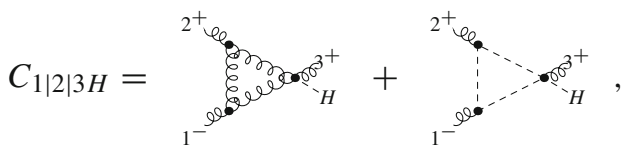

$c_{1|2| 3 H ; 0}=0$,

$c_{1|2| 3 H ; 0}=0$;



$c_{12 \mid 3 H ; 0}=0$,

$c_{12 \mid 3 H ; 2}=0$.

The cut $C_{123 \mid H}$ does not give any contribution. The remaining coefficients are collected in Appendix G. The oneloop amplitude can be obtained by using the coefficients collected in Eqs. (43) and (G.25) and the decomposition (D.13). The result agrees with the literature [63].

\section{Generalized open loop}

The FDF of $d$-dimensional one-loop amplitudes is compatible with methods generating recursively the integrands of one-loop amplitudes $[64,65]$ and leads to the complete reconstruction of the numerator of Feynman integrands as a polynomial in the loop variables, $\ell^{\nu}$ and $\mu$. Our scheme allows for a generalization of the current implementations of these techniques [43-45]. Indeed, currently the latter can reconstruct only the four-dimensional part the numerator of the integrands, which is polynomial in $\ell^{v}$ only. In the following we focus on the generalization of the open-loop technique [43] within the FDF scheme.

Tree-level and one-loop amplitudes, $\mathcal{M}$ and $\delta \mathcal{M}$, can be obtained as a sum of Feynman diagrams

$\mathcal{M}=\sum_{\text {diag }} \mathcal{M}^{\text {(diag) }}, \quad \delta \mathcal{M}=\sum_{\text {diag }} \delta \mathcal{M}^{(\mathrm{diag})}$.
The color factor $\mathcal{C}$ and the $(-2 \epsilon)$-SRs term $\mathcal{T}$ factorize, thus they can be stripped off each diagram

$\mathcal{M}^{\text {(diag) }}=\mathcal{C}^{\text {(diag) }} \mathcal{A}^{\text {(diag) }}$
$\delta \mathcal{M}^{\text {(diag) }}=\mathcal{C}^{\text {(diag) }} \mathcal{T}^{\text {(diag) }} \mathcal{A}^{\text {(diag) }}$.

The color structures are computed once, as described in [43]. The computation of the $(-2 \epsilon)$-SRs prefactors $\mathcal{T}$ turns out to be even easier, since they enter only in the one-loop diagrams and can be computed once and for all. In the 't HooftFeynman gauge they can be either 0 or 1 .

The recursive construction of the color-stripped tree-level diagrams, $\mathcal{A}^{\text {(diag) }}$, is not affected by the new Feynman particles and Feynman rules, which enter at loop-level only.

The one-loop color-stripped diagram $\delta \mathcal{A}^{\text {(diag) }}$, characterized by a given topology $\mathcal{I}_{n}$, is constructed by $n$ tree-level topologies $i_{1}, \ldots, i_{n}$, connected to the loop. The numerator of the one-loop diagram can be expressed as

$\mathcal{N}\left(\mathcal{I}_{n}, \ell, \mu\right)=\sum_{j=0}^{R} \sum_{a=0}^{R-j} \mathcal{N}_{v_{1} \ldots v_{j}}^{[a]}\left(\mathcal{I}_{n}\right) \ell^{\nu_{1}} \ldots \ell^{\nu_{j}} \mu^{a}$

where $R$ is its rank. The diagram is obtained by performing the integration over the $d$-dimensional loop momentum:

$\delta \mathcal{A}^{\text {(diag) }}=\sum_{j=0}^{R} \sum_{a=0}^{R-j} \mathcal{N}_{\nu_{1} \ldots \nu_{j}}^{[a]}\left(\mathcal{I}_{n}\right) I_{n}^{\nu_{1} \ldots v_{j}}\left[\mu^{a}\right]$

where

$I_{n}^{v_{1} \ldots v_{j}}\left[\mu^{a}\right] \equiv \int d^{d} \bar{\ell} \frac{\ell^{v_{1}} \ldots \ell^{v_{j}} \mu^{a}}{D_{0} \ldots D_{n-1}}$.

The starting point of the open-loop technique is to cut a propagator and to remove the denominators. The open numerator can be expressed in terms of the tree-level topology $i_{n}$ and a one-loop topology $\mathcal{I}_{n-1}$ :

$$
\begin{aligned}
& \mathcal{N}^{\beta}{ }_{\alpha}\left(\mathcal{I}_{n}, \ell, \mu\right) \\
& \quad=X_{\gamma \delta}^{\beta}\left(\mathcal{I}_{n}, i_{n}, \mathcal{I}_{n-1}\right) \mathcal{N}_{\alpha}^{\gamma}\left(\mathcal{I}_{n-1}, \ell, \mu\right) \omega^{\delta}\left(i_{n}\right)
\end{aligned}
$$

where $\omega^{\delta}$ is the expression related to the tree-level topology $i_{n}$. The vertices $X_{\gamma \delta}^{\beta}$ are obtained by the FDF Feynman rules, Eq. (131), and they can be written as follows:

$X_{\gamma \delta}^{\beta}=Y_{\gamma \delta}^{\beta}+\ell^{\nu} Z_{v ; \gamma \delta}^{\beta}+\mu W_{\gamma \delta}^{\beta}$.

Therefore the tensor coefficients of the covariant decomposition

$\mathcal{N}_{\alpha}^{\beta}\left(\mathcal{I}_{n}, \ell, \mu\right)=\sum_{j=0}^{R} \sum_{a=0}^{R-j} \mathcal{N}_{v_{1} \ldots v_{j} ; \alpha}^{[a] \beta}\left(\mathcal{I}_{n}\right) \ell^{\nu_{1}} \ldots \ell^{\nu_{j}} \mu^{a}$ 
are obtained by the recursive relation

$$
\begin{aligned}
\mathcal{N}_{\nu_{1} \ldots v_{j} ; \alpha}^{[a] \beta}\left(\mathcal{I}_{n}\right)= & {\left[Y_{\gamma \delta}^{\beta} \mathcal{N}_{\nu_{1} \ldots v_{j} ; \alpha}^{[a] \gamma}\left(\mathcal{I}_{n-1}\right)\right.} \\
& +Z_{\nu_{1} ; \gamma \delta}^{\beta} \mathcal{N}_{\nu_{2} \ldots v_{j} ; \alpha}^{[a] \gamma}\left(\mathcal{I}_{n-1}\right) \\
& \left.+W_{\gamma \delta}^{\beta} \mathcal{N}_{\nu_{1} \ldots v_{j} ; \alpha}^{[a-1] \gamma}\left(\mathcal{I}_{n-1}\right)\right] \omega^{\delta}\left(i_{n}\right) .
\end{aligned}
$$

The recursive generation of integrands within the FDF can be suitably combined with public codes like SAMURAI [66] and NiNJA $[67,68]$, which can reduce integrands keeping the full dependence on the loop variables $\ell^{v}$ and $\mu$. Moreover, it can improve the generation of the $d$-dimensional integrands performed by the packages GOSAM [69] and FORMCALC [49]. The latter are public codes dedicated to the automatic evaluation of one-loop multi-particle scattering amplitudes, and they already support the FDH regularization scheme.

\section{Conclusions}

We introduced a four-dimensional formulation (FDF) of the $d$-dimensional regularization of one-loop scattering amplitudes. Within our FDF, particles that propagate inside the loop are represented by massive particles regularizing the divergences. Their interactions are described by generalized four-dimensional Feynman rules. They include selection rules accounting for the regularization of the amplitudes. In particular, massless spin-1 particles in $d$-dimensions were represented in four dimensions by a combination of massive spin-one particle and a scalar particle. Fermions in $d$ dimensions were represented by four-dimensional fermions obeying the Dirac equation for tardyonic particles. The integrands of one-loop amplitudes in the FDF and in the FDH scheme differ by spurious terms which vanish upon integration over the loop momentum. Therefore the two schemes are equivalent.

In the FDF, the polarization and helicity states of the particles inside the loop admit an explicit four-dimensional representation, allowing for a complete, four-dimensional, unitarity-based construction of $d$-dimensional amplitudes. The application of generalized-unitarity methods within the FDF has been described in detail by computing the NLO QCD corrections to helicity amplitudes of the processes $g g \rightarrow q \bar{q}$ and $g g \rightarrow g H$.

Mutual cancelations among the contributions of the longitudinal gluons and the ones of the scalar particles suggest a connection among them that deserves further investigations.

The FDF Feynman rules are compatible with methods generating recursively the integrands of one-loop amplitudes. In this context we have proposed a generalization to the openloop method, which allows for a complete reconstruction of the integrand, currently limited to four dimensions only.
The FDF approach is suitable for analytic as well as numerical implementation. Its main asset is the use of purely four-dimensional ingredients for the complete reconstruction of dimensionally regulated one-loop amplitudes. We plan to investigate its applicability beyond one loop. In particular we aim at using explicit four-dimensional representations to avoid the complications emerging from the formal manipulations of the $(d-4)$-dimensional degrees of freedom.

Acknowledgments We wish to thank Francesco Buciuni for crosschecking parts of the results. A.R.F. and W.J.T. thank the Max-PlanckInstitute for Physics in Munich for the kind hospitality at several stages of this project. For the same reasons, E.M. wishes to thank the Department of Mathematics and Physics of the University of Salento. A.R.F. is partially supported by the UNAL-DIB Grant No. 20629 of the "Convocatoria del programa nacional de proyectos para el fortalecimiento de la investigaciòn, la creaciòn y la innovaciòn en posgrados de la Universidad Nacional de Colombia 2013-2015”. The work of P.M. is supported by the Alexander von Humboldt Foundation, in the framework of the Sofja Kovaleskaja Award Project "Advanced Mathematical Methods for Particle Physics", endowed by the German Federal Ministry of Education and Research. W.J.T. is supported by Fondazione Cassa di Risparmio di Padova e Rovigo (CARIPARO).

Open Access This article is distributed under the terms of the Creative Commons Attribution License which permits any use, distribution, and reproduction in any medium, provided the original author(s) and the source are credited.

Funded by $\mathrm{SCOAP}^{3}$ / License Version CC BY 4.0.

\section{Appendix A: One-loop equivalence}

In this appendix we show that, at one loop, the FDH scheme defined by Eqs. (2)-(5b) is equivalent to the one defined by Eqs. (2)-(5a) and (10).

In the two approaches the only differences may arise from the manipulations of the $-2 \epsilon$ components of the Dirac matrices contracted among each others. Therefore potential differences in their predictions can only be rational contributions of divergent diagrams involving at least an open fermion line. The loop-dependent part of the integrand of a one-loop diagram is a sum of integrands of the type

$\mathcal{I}_{r, a, k} \equiv \frac{\ell^{\mu_{1}} \ldots \ell^{\mu_{r}}\left(\mu^{2}\right)^{a}}{D_{i_{1}} \ldots D_{i_{k}}}, \quad D_{j} \equiv\left(\ell+p_{j}\right)^{2}-m_{j}-\mu^{2}$.

An integrand $\mathcal{I}_{r, a, k}$ leads to a divergent integral if it satisfies the conditions

$4+r+2 a-2 k \geq 0$

At one loop in QCD the diagrams involving at least an open fermion line and integrands fulfilling the conditions (A.2) are




For these diagrams, the numerators obtained by using the two schemes differ by terms of the type

$$
\begin{aligned}
& \ldots \tilde{\gamma}^{\alpha}(\ell+\tilde{\ell}+m) \tilde{\gamma}_{\alpha} \ldots, \\
& \ldots \tilde{\gamma}^{\alpha}(\ell+\tilde{\ell}+m) \gamma^{\mu}(\ell+\not p+\tilde{\ell}+m) \tilde{\gamma}_{\alpha} \ldots,
\end{aligned}
$$

where "..." represent four-dimensional spinorial objects. In the FDH scheme it is easy to show that the terms (A.3) vanish in the $d_{s} \rightarrow 4$ limit, while in the other scheme they vanish as a consequence of Eq. (10). Therefore the two sets of prescriptions lead to the same integrand.

The FDF fulfills the prescriptions (2)-(5a) and (10), thus, at one loop, it leads to the same amplitudes of the FDH scheme.

\section{Appendix B: Proof of the completeness relations}

In this appendix we show that the generalized spinors (20) fulfill the completeness relation (19). For later convenience we define the chirality projectors

$\omega_{ \pm}=\frac{\mathbb{I} \pm \gamma^{5}}{2}$

and we show that:

$$
\begin{aligned}
& \frac{\left.\mid q_{\ell}\right]\left[\ell^{b}|-| l^{b}\right]\left[q_{\ell} \mid\right.}{\left[\ell^{b} q_{\ell}\right]}=\frac{\left.\mid q_{\ell}\right]\left\langle q_{\ell} \ell^{b}\right\rangle\left[\ell^{b}|+| \ell^{b}\right]\left\langle\ell^{b} q_{\ell}\right\rangle\left[q_{\ell} \mid\right.}{2 \ell^{b} \cdot q_{\ell}} \\
& =\frac{\left.\left(\mid q_{\ell}\right]\left\langle q_{\ell}\right|\right)\left(\left|\ell^{b}\right\rangle\left[\ell^{b} \mid\right)+\left(\mid \ell^{b}\right]\left\langle\ell^{b}\right|\right)\left(\left|q_{\ell}\right\rangle\left[q_{\ell} \mid\right)\right.}{2 \ell^{b} \cdot q_{\ell}} \\
& =\frac{\omega_{-} q_{\ell} \omega_{+} \ell^{b}+\omega_{-} \ell^{b} \omega_{+} q_{\ell}}{2 \ell^{b} \cdot q_{\ell}} \\
& =\frac{\omega_{-}^{2}\left\{q_{\ell} \ell^{b}\right\}}{2 \ell^{b} \cdot q_{\ell}}=\omega_{-}
\end{aligned}
$$

and similarly

$$
\frac{\left|\ell^{b}\right\rangle\left\langle q_{\ell}|-| q_{\ell}\right\rangle\left\langle\ell^{b}\right|}{\left\langle q_{\ell} \ell^{b}\right\rangle}=\omega_{+} .
$$

Using Eqs. (B.5) we get

$$
\begin{aligned}
\sum_{\lambda= \pm} u_{\lambda}(\ell) \bar{u}_{\lambda}(\ell)= & \left.\left(\left|\ell^{b}\right\rangle+\frac{(m-i \mu)}{\left[\ell^{b} q_{\ell}\right]} \mid q_{\ell}\right]\right) \\
& \times\left(\left[\ell^{b} \mid+\frac{(m+i \mu)}{\left\langle q_{\ell} \ell^{b}\right\rangle}\left\langle q_{\ell}\right|\right)\right. \\
& \left.+\left(\mid \ell^{b}\right]+\frac{(m+i \mu)}{\left\langle\ell^{b} q_{\ell}\right\rangle}\left|q_{\ell}\right\rangle\right) \\
& \times\left(\left\langle\ell^{b}\right|+\frac{(m-i \mu)}{\left[q_{\ell} \ell^{b}\right]}\left[q_{\ell} \mid\right)\right. \\
= & \ell^{b}+\frac{m^{2}+\mu^{2}}{2 \ell^{b} \cdot q_{\ell}} q_{\ell} \\
& +(m-i \mu) \frac{\left.\mid q_{\ell}\right]\left[\ell^{b}|-| \ell^{b}\right]\left[q_{\ell} \mid\right.}{\left[\ell^{b} q_{\ell}\right]}
\end{aligned}
$$

$$
\begin{aligned}
& \quad+(m+i \mu) \frac{\left|\ell^{b}\right\rangle\left\langle q_{\ell}|-| q_{\ell}\right\rangle\left\langle\ell^{b}\right|}{\left\langle q_{\ell} \ell^{b}\right\rangle} \\
& \text { Eq. (B.5) } \ell^{b}+\frac{m^{2}+\mu^{2}}{2 \ell^{b} \cdot q_{\ell}} q_{\ell} \\
& \quad+(m-i \mu) \omega_{-}+(m+i \mu) \omega_{+} \\
& \stackrel{\text { Eq. (17) }}{=} \ell+i \mu \gamma^{5}+m .
\end{aligned}
$$

\section{Appendix C: Color-ordered Feynman rules}

In the FDF, the $d$-dimensional color-ordered Feynman rules collected in [70] become:

$\underset{\alpha}{\stackrel{k}{k} \text {.uе }_{\beta}}=-i \frac{g^{\alpha \beta}}{k^{2}-\mu^{2}+i 0}, \quad$ (gluon),

$\stackrel{k}{\cdot-{ }_{A}}=-i \frac{G^{A B}}{k^{2}-\mu^{2}+i 0}, \quad$ (scalar),

$\stackrel{k}{\longrightarrow} \cdot=i \frac{\not k+i \mu \gamma^{5}+m}{k^{2}-m^{2}-\mu^{2}+i 0} . \quad$ (fermion),

$\operatorname{lum}_{1, \alpha}^{2, \beta} \log _{3, \gamma}^{\sigma_{2}}=\frac{i}{\sqrt{2}}\left[g_{\alpha \beta}\left(k_{1}-k_{2}\right)_{\gamma}+g_{\beta \gamma}\left(k_{2}-k_{3}\right)_{\alpha}\right.$

$\left.+g_{\gamma \alpha}\left(k_{3}-k_{1}\right)_{\beta}\right]$

$\underset{1, \alpha}{2, B} \operatorname{coc}_{3, C}=\frac{i}{\sqrt{2}}\left(k_{2}-k_{3}\right)_{\alpha} G^{B C}$,

$\underset{1, \alpha}{2, B,-,} \log _{3, \gamma}= \pm \frac{i}{\sqrt{2}} g_{\alpha \gamma}(i \mu) Q^{B}$

$\left(\tilde{k}_{1}=0, \quad \tilde{k}_{3}= \pm \tilde{\ell}\right)$

$\operatorname{lum}_{3, C}^{2, \beta} \cos ^{6^{6}}=\mp \frac{i}{\sqrt{2}} g_{\alpha \beta}(i \mu) Q^{C}$

$$
\left(\tilde{k}_{1}=0, \quad \tilde{k}_{3}= \pm \tilde{\ell}\right)
$$

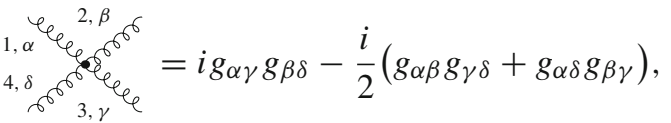

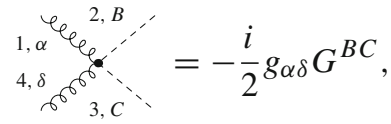

$\overrightarrow{1} \cdot 0_{3}^{2, \beta}=-\frac{i}{\sqrt{2}} \gamma^{\beta}$,

$\overbrace{3}^{2, \beta} \cdot \sigma^{6^{6}}=\frac{i}{\sqrt{2}} \gamma^{\beta}$, 


$$
\begin{aligned}
& =-\frac{i}{\sqrt{2}} \gamma^{5} \Gamma^{B}, \\
1 & =\frac{i}{\sqrt{2}} \gamma^{5} \Gamma^{B},
\end{aligned}
$$

The color-ordered Feynman rules describing the interaction among an external Higgs boson and gluons in the infinite top-mass limit are given by

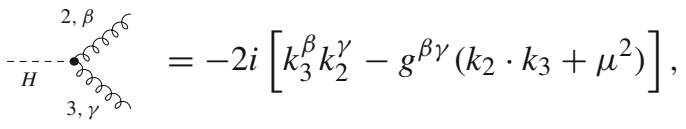

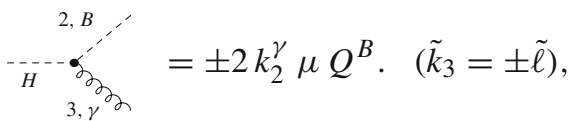

$$
\begin{aligned}
& { }_{3, C}^{2, \beta} \cdot \sigma^{6}= \pm 2 k_{3}^{\beta} \mu Q^{C}, \quad\left(\tilde{k}_{2}= \pm \tilde{\ell}\right) \\
& \stackrel{\ddots}{2, B}=-2 i\left[\mu^{2} Q^{B} Q^{C}-G^{B C}\left(k_{2} \cdot k_{3}+\mu^{2}\right)\right],
\end{aligned}
$$

$$
\text { ר, }
$$



In the Feynman rules (C.7), (C.8) all the momenta are outgoing. The terms $\mu^{2}$ appearing in the the propagators (C.7a)(C.7c) enter only if the corresponding momentum $k$ is $d$ dimensional, i.e. only if $k$ contains the loop momentum $\bar{\ell}$. In the vertices $(\mathrm{C} .7 \mathrm{f}),(\mathrm{C} .7 \mathrm{~g})$ the momentum $k_{1}$ is fourdimensional while the other two are $d$-dimensional. For these vertices the overall sign depend on which of the combinations (14) is present in the vertex. Similarly the overall sign of the Feynman rules (C.8b), (C.8c) and (C.8g) depend on the flow of the loop momentum $\bar{\ell}$. As already mentioned each cut scalar propagator carries a $(-2 \epsilon)$-SRs factor of the type

${ }_{A} \cdot{ }_{B}=\hat{G}^{A B}$,

where $\hat{G}^{A B}$ is defined in Eq. (29).

\section{Appendix D: One-loop amplitudes}

In this section we present the decomposition of the one-loop amplitudes presented in Sects. 4-6 in terms of the MIs. We consider the one-loop four-point amplitudes with four outgoing massless particles,

$0 \rightarrow 1\left(p_{1}\right) 2\left(p_{2}\right) 3\left(p_{3}\right) 4\left(p_{4}\right)$,

where $p_{i}$ is the momentum of the particle $i$. In general, a massless four-point one-loop amplitude can be decomposed in terms MIs, as follows:

$$
\begin{aligned}
A_{4}= & \frac{1}{(4 \pi)^{2-\epsilon}}\left[c_{1|2| 3 \mid 4 ; 0} I_{1|2| 3 \mid 4}+\left(c_{12|3| 4 ; 0} I_{12|3| 4}\right.\right. \\
& \left.+c_{1|2| 34 ; 0} I_{1|2| 34}+c_{1|23| 4 ; 0} I_{1|23| 4}+c_{2|3| 41 ; 0} I_{2|3| 41}\right) \\
& +\left(c_{12 \mid 34 ; 0} I_{12 \mid 34}+c_{23 \mid 41 ; 0} I_{23 \mid 41}\right) \\
& +c_{1|2| 3 \mid 4 ; 4} I_{1|2| 3 \mid 4}\left[\mu^{4}\right]+\left(c_{12|3| 4 ; 2} I_{12 \mid 34}\left[\mu^{2}\right]\right. \\
& +c_{1|2| 34 ; 2} I_{1|2| 34}\left[\mu^{2}\right]+c_{1|23| 4 ; 2} I_{1|23| 4}\left[\mu^{2}\right] \\
& \left.+c_{2|3| 41 ; 2} I_{2|3| 41}\left[\mu^{2}\right]\right) \\
& \left.\left.+\left(c_{12 \mid 34 ; 2} I_{12 \mid 34}\left[\mu^{2}\right]+c_{23 \mid 41 ; 2} I_{23 \mid 41}\left[\mu^{2}\right]\right)\right] . \quad \text { D. } 11\right)
\end{aligned}
$$

We consider also the process involving three gluons, 1, 2, 3, and a Higgs boson, $H$,

$0 \rightarrow 1\left(p_{1}\right) 2\left(p_{2}\right) 3\left(p_{3}\right) H\left(p_{H}\right)$

in the large top-mass limit $[71,72]$. The one-loop amplitude for this process is decomposed as follows:

$$
\begin{aligned}
A_{4, H}= & \frac{1}{(4 \pi)^{2-\epsilon}}\left[\left(c_{1|2| 3 \mid H ; 0} I_{1|2| 3 \mid H}+c_{1|2| H \mid 3 ; 0} I_{1|2| H \mid 3}\right.\right. \\
& \left.+c_{1|H| 2 \mid 3 ; 0} I_{1|H| 2 \mid 3}\right)+\left(c_{12|3| H ; 0} I_{12|3| H}\right. \\
& +c_{12|H| 3 ; 0} I_{12|H| 3}+c_{1|23| H ; 0} I_{1|23| H} \\
& +c_{1|H| 23 ; 0} I_{1|H| 23}+c_{2|H| 31 ; 0} I_{2|H| 31} \\
& +c_{H|2| 31 ; 0} I_{H|2| 31}+c_{1|2| 3 H ; 0} I_{1|2| 3 H} \\
& \left.+c_{1|2 H| 3 ; 0} I_{1|2 H| 3}+c_{1 H|2| 3 ; 0} I_{1 H|2| 3}\right) \\
& +\left(c_{12 \mid 3 H ; 0} I_{12 \mid 3 H}+c_{23 \mid H 1 ; 0} I_{23 \mid H 1}\right. \\
& \left.+c_{H 2 \mid 31 ; 0} I_{H 2 \mid 31}\right)+c_{123 \mid H ; 0} I_{123 \mid H} \\
& +\left(c_{1|2| 3 \mid H ; 4} I_{1|2| 3 \mid H}\left[\mu^{4}\right]\right. \\
& +c_{1|2| H \mid 3 ; 4} I_{1|2| H \mid 3}\left[\mu^{4}\right] \\
& \left.+c_{1|H| 2 \mid 3 ; 4} I_{1|H| 2 \mid 3}\left[\mu^{4}\right]\right) \\
& +\left(c_{12|3| H ; 2} I_{12|3| H}\left[\mu^{2}\right]+c_{12|H| 3 ; 2} I_{12|H| 3}\left[\mu^{2}\right]\right. \\
& +c_{1|23| H ; 2} I_{1|23| H}\left[\mu^{2}\right]+c_{1|H| 23 ; 2} I_{1|H| 23}\left[\mu^{2}\right] \\
& +c_{2|H| 31 ; 2} I_{2|H| 31}\left[\mu^{2}\right]+c_{H|2| 31 ; 2} I_{H|2| 31}\left[\mu^{2}\right]
\end{aligned}
$$




$$
\begin{aligned}
& +c_{1|2| 3 H ; 2} I_{1|2| 3 H}\left[\mu^{2}\right]+c_{1|2 H| 3 ; 2} I_{1|2 H| 3}\left[\mu^{2}\right] \\
& \left.+c_{1 H|2| 3 ; 2} I_{1 H|2| 3}\left[\mu^{2}\right]\right) \\
& +\left(c_{12 \mid 3 H ; 2} I_{12 \mid 3 H}\left[\mu^{2}\right]+c_{23 \mid H 1 ; 2} I_{23 \mid H 1}\left[\mu^{2}\right]\right. \\
& \left.\left.+c_{H 2 \mid 31 ; 2} I_{H 2 \mid 31}\left[\mu^{2}\right]+c_{123 \mid H ; 2} I_{123 \mid H}\left[\mu^{2}\right]\right)\right] .
\end{aligned}
$$

The coefficients $c$ 's entering in the decompositions (D.11) and (D.13) can be obtained by using the generalized-unitarity techniques for quadruple [5,73], triple [73-75], and double [76-78] cuts. We observe that single-cut techniques [79$81]$ are not needed because of the absence of ( $d$-dimensional) massive particles in the loop.

\section{Appendix E: Three-point amplitudes}

The tree-level three-point amplitudes relevant for the computation presented in Sect. 4 are the ones involving either three gluons or two scalars and one gluon. The tree-level amplitudes with two gluons and one scalar should be included as well but they are not needed since their cut diagrams vanish because of the $(-2 \epsilon)$-SRs, cfr. Sect. 4 . The tree-level amplitudes are computed by using the color-ordered Feynman rules collected in Appendix C.

The general expression of the three-point all-gluon amplitude is given by

$$
\begin{aligned}
& \underset{2^{\lambda_{2}} \log _{3_{3}}^{\boldsymbol{\lambda}_{3}}}{\mathbf{1}^{\lambda_{1}} \sigma^{\sigma}}=\frac{i g}{\sqrt{2}}\left[g^{\mu \nu}(\mathbf{1}-2)^{\sigma}+g^{\nu \sigma}(2-\mathbf{3})^{\mu}\right. \\
& \left.+g^{\sigma \mu}(\mathbf{3}-\mathbf{1})^{\nu}\right] \varepsilon_{\mu}^{\lambda_{1}}(\mathbf{1}) \varepsilon_{v}^{\lambda_{2}}\left(2, r_{2}\right) \varepsilon_{\sigma}^{\lambda_{3}}(\mathbf{3}) \text {. }
\end{aligned}
$$

Generalized massive momenta, carrying dependence on $\mu$, are denoted by a bold font, and the polarization of the particle will be the superscript of the corresponding momentum. The momenta are outgoing,

$\mathbf{1}+2+\mathbf{3}=0$

and in general $\hat{q}_{\mathbf{1}}$ and $\hat{q}_{\mathbf{3}}$ can be chosen to be proportional,

$\hat{q}_{3}=\xi \hat{q}_{\mathbf{1}}$.

Moreover, the spinors associated to the momenta $\mathbf{j}^{\mathrm{b}}$ and $\hat{q}_{\mathbf{j}}$ are such that

$$
\left\langle\mathbf{j}^{b} \mid \hat{q}_{\mathbf{j}}\right\rangle=\left[\hat{q}_{\mathbf{j}} \mid \mathbf{j}^{\mathrm{b}}\right]=\mu, \quad \mathbf{j}=\mathbf{1}, \mathbf{3}
$$

The polarization vector associated to a massless momentum $k$ is defined as [70] $\varepsilon_{+}^{\mu}\left(k, r_{k}\right)=\frac{\left\langle r_{k}\left|\gamma^{\mu}\right| k\right]}{\sqrt{2}\left\langle r_{k} k\right\rangle}$,

$\varepsilon_{-}^{\mu}\left(k, r_{k}\right)=-\frac{\left[r_{k}\left|\gamma^{\mu}\right| k\right\rangle}{\sqrt{2}\left[r_{k} k\right]}$,

in terms of an arbitrary reference spinor $r_{k}$. We observe that the amplitude (E.14) is independent of the choice of $r_{2}$. The proof proceeds along the lines of a similar proof presented in [82]. A change in the reference momentum shifts the amplitude (E.14) by an amount proportional to

$$
\begin{aligned}
& {\left[g^{\mu \nu}(\mathbf{1}-2)^{\sigma}+g^{v \sigma}(2-\mathbf{3})^{\mu}+g^{\sigma \mu}(\mathbf{3}-\mathbf{1})^{\nu}\right]} \\
& \varepsilon_{\mu}^{\lambda_{1}}(\mathbf{1}) 2_{\nu} \varepsilon_{\sigma}^{\lambda_{3}}(\mathbf{3}),
\end{aligned}
$$

which vanishes owing to momentum conservation, Eq. (E.15), and to the transversality condition (28).

The explicit expressions of the polarized amplitudes in the FDF are:

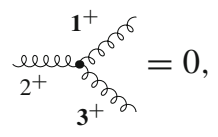

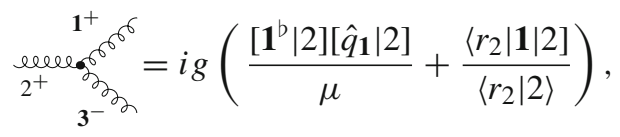

$$
\begin{aligned}
& \operatorname{2ul}_{2^{+}}^{1_{3^{+}}^{0}} \cos ^{6^{6}}=0
\end{aligned}
$$



$$
\begin{aligned}
& \operatorname{lelel}_{2^{+}}^{\mathbf{1}_{3_{2}^{-}}^{6^{6}}}=i g \frac{\left[\hat{q}_{\mathbf{1}} \mid 2\right]\left[\hat{q}_{\mathbf{3}} \mid 2\right]\left\langle\mathbf{1}^{b} \mid \mathbf{3}^{b}\right\rangle}{\mu^{2}}, \\
& \operatorname{2uleg}_{2_{2}^{+}}^{\mathbf{1}^{0} \sigma^{6^{6}}}=-i g \frac{\left\langle r_{2}|\mathbf{1}| 2\right]}{\left\langle r_{2} \mid 2\right\rangle} \\
& {\left[1-\frac{(1+\xi)}{\xi \mu^{2}}\left((1+\xi) \mu^{2}+\xi\left\langle\hat{q}_{1}|2| \hat{q}_{1}\right]\right)\right] \text {. }}
\end{aligned}
$$

The three-point amplitude involving a gluon and two scalars is

$$
\begin{aligned}
\varkappa_{2^{+}}^{1,-} & =\frac{i g}{\sqrt{2}}(\mathbf{3}-\mathbf{1})^{\mu} \varepsilon_{\mu}^{+}\left(2, r_{2}\right) G^{A B} \\
& =-i g \frac{\left\langle r_{2}|\mathbf{1}| 2\right]}{\left\langle r_{2} \mid 2\right\rangle} G^{A B} .
\end{aligned}
$$




\section{Appendix F: Results for the ggq $\bar{q}$ right-turning amplitude}

In this appendix we collect the coefficients entering the decomposition (D.11) of the right-turning amplitude $A_{4}^{\mathrm{R}}$. The corresponding cuts are shown as well. The quadruple cut is given by



$c_{1|2| 3 \mid 4 ; 0}^{[\mathrm{R}]}=-\frac{1}{2} A_{4}^{\text {tree }} \frac{s_{12}^{3}}{s_{13}^{3}} s_{12} s_{14}$,

$c_{1|2| 3 \mid 4 ; 4}^{[\mathrm{R}]}=0$.

The first helicity configuration contributes only to the cutconstructible part while the second one cancels against the box with internal scalars. The triple cuts are given by

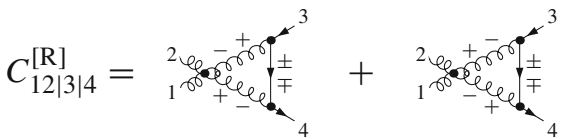

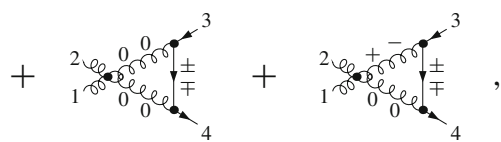

$$
\begin{aligned}
& c_{12|3| 4 ; 0}^{[\mathrm{R}]}=-\frac{1}{2} A_{4}^{\text {tree }}\left(2+\frac{s_{12}^{3}}{s_{13}^{3}}\right) s_{12}, \\
& c_{12|3| 4 ; 2}^{[\mathrm{R}]}=-\frac{1}{2} A_{4}^{\mathrm{tree}}\left(1+\frac{s_{14}^{2}}{s_{13}^{2}}\right) \text {; }
\end{aligned}
$$

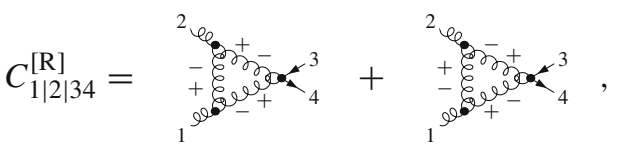



$$
\begin{aligned}
& c_{1|23| 4 ; 0}^{[\mathrm{R}]}=-\frac{1}{2} A_{4}^{\text {tree }} \frac{s_{12}^{3}}{s_{13}^{3}} s_{14}, \\
& c_{1|23| 4 ; 2}^{[\mathrm{R}]}=-\frac{1}{2} A_{4}^{\mathrm{tree}} \frac{s_{12} s_{14}}{s_{13}^{2}} \text {; }
\end{aligned}
$$



$c_{2|3| 41 ; 0}^{[\mathrm{R}]}=-\frac{1}{2} A_{4}^{\text {tree }} \frac{s_{12}^{3}}{s_{13}^{3}} s_{14}$,

$c_{2|3| 41 ; 2}^{[\mathrm{R}]}=-\frac{1}{2} A_{4}^{\mathrm{tree}} \frac{s_{12} s_{14}}{s_{13}^{2}}$.

In the case of the cuts $C_{12|3| 4}^{[\mathrm{R}]}$ and $C_{1|2| 34}^{[\mathrm{R}]}$ the first diagram gives contributions to the both cut-constructible and the rational part, while the second one contributes to the rational part only. In the cuts $C_{12|3| 4}^{[\mathrm{R}]}, C_{1|23| 4}^{[\mathrm{R}]}$ and $C_{2|3| 41}^{[\mathrm{R}]}$ the last two diagrams cancel against each other, i.e. the scalar contribution exactly compensates the contribution of the longitudinal polarization of the gluon. The double cuts are

$$
\begin{aligned}
& C_{12 \mid 34}^{[\mathrm{R}]}= \\
& c_{12 \mid 34 ; 0}^{[\mathrm{R}]}=A_{4}^{\text {tree }}\left[\frac{s_{12}}{s_{13}}\left(\frac{s_{14}}{s_{13}}+\frac{3}{2}\right)+\frac{3}{2}\right], \\
& c_{12 \mid 34 ; 2}^{[\mathrm{R}]}=0 ;
\end{aligned}
$$

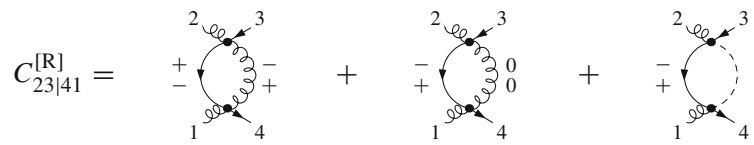

$c_{23 \mid 41 ; 0}^{[\mathrm{R}]}=-A_{4}^{\text {tree }} \frac{s_{12}}{s_{13}}\left(\frac{s_{14}}{s_{13}}+\frac{3}{2}\right)$,

$c_{23 \mid 41 ; 2}^{[\mathrm{R}]}=0$.

For the cut $C_{24}^{[\mathrm{R}]}$, the first diagram contributes to the cutconstructible part only while the second one is canceled by the diagram with an internal scalar.

\section{Appendix G: Coeffcients of the gggH amplitude for the orderings $12 \mathrm{H} 3$ and $1 \mathrm{H} 23$}

The coefficients entering the decomposition (D.13) and corresponding to the orderings $12 \mathrm{H} 3$ and $1 \mathrm{H} 23$ reads as follows:



$c_{1|2| H \mid 3 ; 0}=-\frac{1}{2} A_{4, H}^{\text {tree }} s_{13} s_{12}$,

$c_{1|2| H \mid 3 ; 4}=0$; 


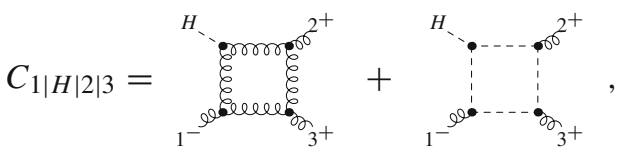

$$
\begin{aligned}
& c_{1|H| 2 \mid 3 ; 0}=-\frac{1}{2} A_{4, H}^{\text {tree }} s_{23} s_{13}, \\
& c_{1|H| 2 \mid 3 ; 4}=0 \text {; }
\end{aligned}
$$



$$
\begin{aligned}
& c_{12|H| 3 ; 0}=\frac{1}{2} A_{4, H}^{\text {tree }}\left(s_{13}+s_{23}\right), \\
& c_{12|H| 3 ; 2}=0 \text {; }
\end{aligned}
$$

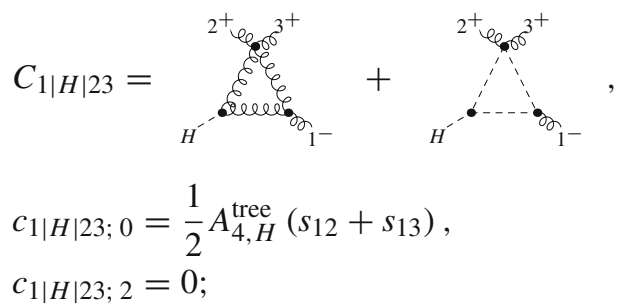

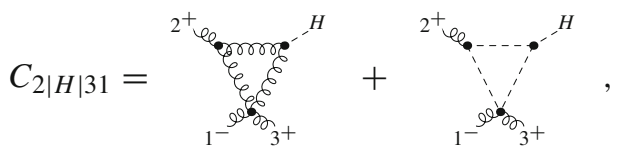$$
c_{2|H| 310}=\frac{1}{2} A_{4, H}^{\text {tree }}\left(s_{12}+s_{23}\right),
$$$$
c_{2|H| 312}=0 \text {; }
$$

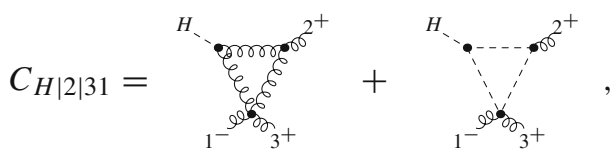$$
c_{H|2| 31 ; 0}=\frac{1}{2} A_{4, H}^{\text {tree }}\left(s_{12}+s_{23}\right),
$$$$
c_{H|2| 31 ; 2}=0 \text {; }
$$

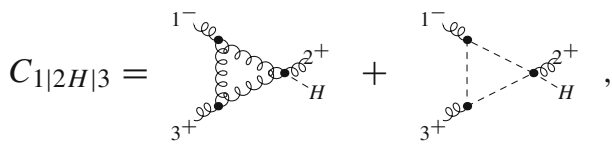

$c_{1|2 H| 3 ; 0}=0$,

$c_{1|2 H| 3 ; 0}=0$;

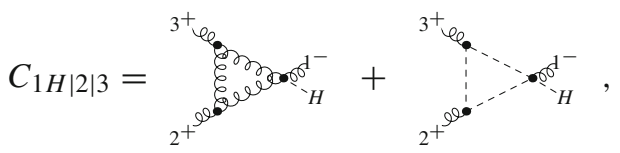

$c_{1 H|2| 3 ; 0}=0$,

$c_{1 H|2| 3 ; 2}=-2 A_{4, H}^{\text {tree }} \frac{s_{12} s_{13}}{s_{23}^{2}} ;$

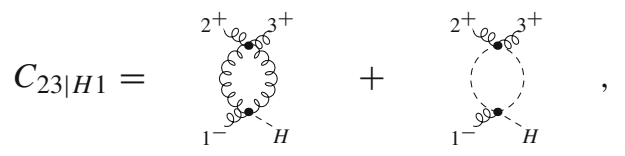

$c_{23 \mid H 1 ; 0}=0$,

$c_{23 \mid H 1 ; 2}=4 A_{4, H}^{\text {tree }} \frac{s_{12} s_{13}}{s_{23}^{3}} ;$

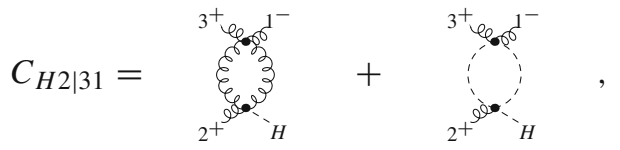

$c_{H 2 \mid 31 ; 0}=0$,

$c_{H 2 \mid 31 ; 2}=0$. amplitudes to scalar integrals at the integrand level. Nucl. Phys. B 763, 147-169 (2007). hep-ph/0609007

7. Y. Zhang, Integrand-level reduction of loop amplitudes by computational algebraic geometry methods. JHEP 1209, 042 (2012). arXiv: 1205.5707

8. P. Mastrolia, E. Mirabella, G. Ossola, T. Peraro, Scattering amplitudes from multivariate polynomial division. Phys. Lett. B 718, 173-177 (2012). arXiv:1205.7087

9. D.A. Kosower, K.J. Larsen, Maximal unitarity at two loops. Phys. Rev. D 85, 045017 (2012). arXiv:1108.1180

10. K.J. Larsen, Global poles of the two-loop six-point $N=4$ SYM integrand. Phys. Rev. D 86, 085032 (2012). arXiv: 1205.0297

11. S. Caron-Huot, K.J. Larsen, Uniqueness of two-loop master contours. JHEP 1210, 026 (2012). arXiv: 1205.0801

12. L.F. Alday, R. Roiban, Scattering amplitudes, Wilson loops and the string/gauge theory correspondence. Phys. Rep. 468, 153-211 (2008). arXiv:0807.1889

13. R. Britto, Loop amplitudes in gauge theories: modern analytic approaches. J. Phys. A 44, 454006 (2011). arXiv:1012.4493. 34 pages. Invited review for a special issue of J. Phys. A devoted to 'Scattering Amplitudes in Gauge Theories'

14. J.M. Henn, Dual conformal symmetry at loop level: massive regularization. J. Phys. A 44, 454011 (2011). arXiv:1103.1016

15. Z. Bern, Y.-T. Huang, Basics of generalized unitarity. J. Phys. A 44, 454003 (2011). arXiv:1103.1869

16. J.J.M. Carrasco, H. Johansson, Generic multiloop methods and application to $N=4$ super-Yang-Mills. J. Phys. A 44, 454004 (2011). arXiv:1103.3298 
17. L.J. Dixon, Scattering amplitudes: the most perfect microscopic structures in the universe. J. Phys. A 44, 454001 (2011). arXiv: 1105.0771

18. R. Ellis, Z. Kunszt, K. Melnikov, G. Zanderighi, One-loop calculations in quantum field theory: from Feynman diagrams to unitarity cuts. arXiv: 1105.4319

19. H. Ita, Susy theories and QCD: numerical approaches. J. Phys. A 44, 454005 (2011). arXiv:1109.6527

20. G. Ossola, C.G. Papadopoulos, R. Pittau, Numerical evaluation of six-photon amplitudes. JHEP 0707, 085 (2007). arXiv:0704.1271

21. P. Mastrolia, G. Ossola, On the integrand-reduction method for two-loop scattering amplitudes. JHEP 1111, 014 (2011). arXiv:1107.6041

22. S. Badger, H. Frellesvig, Y. Zhang, Hepta-cuts of two-loop scattering amplitudes. JHEP 1204, 055 (2012). arXiv:1202.2019

23. P. Mastrolia, E. Mirabella, G. Ossola, T. Peraro, Multiloop integrand reduction for dimensionally regulated amplitudes. Phys. Lett. B 727, 532-535 (2013). arXiv: 1307.5832

24. R. Pittau, A four-dimensional approach to quantum field theories. JHEP 1211, 151 (2012). arXiv: 1208.5457

25. A.M. Donati, R. Pittau, Gauge invariance at work in FDR: $H \rightarrow$ $\gamma \gamma$. JHEP 1304, 167 (2013). arXiv: 1302.5668

26. A.M. Donati, R. Pittau, FDR, an easier way to NNLO calculations: a two-loop case study. arXiv:1311.3551

27. G. Mahlon, One loop multi-photon helicity amplitudes. Phys. Rev. D 49, 2197-2210 (1994). hep-ph/9311213

28. Z. Bern, A.G. Morgan, Massive loop amplitudes from unitarity. Nucl. Phys. B 467, 479-509 (1996). hep-ph/9511336

29. C. Anastasiou, R. Britto, B. Feng, Z. Kunszt, P. Mastrolia, Ddimensional unitarity cut method. Phys. Lett. B 645, 213-216 (2007). hep-ph/0609191

30. C. Anastasiou, R. Britto, B. Feng, Z. Kunszt, P. Mastrolia, Unitarity cuts and reduction to master integrals in d dimensions for one-loop amplitudes. JHEP 0703, 111 (2007). hep-ph/0612277

31. W.T. Giele, Z. Kunszt, K. Melnikov, Full one-loop amplitudes from tree amplitudes. JHEP 0804, 049 (2008). arXiv:0801.2237

32. R. Ellis, W.T. Giele, Z. Kunszt, K. Melnikov, Masses, fermions and generalized $D$-dimensional unitarity. Nucl. Phys. B 822, 270-282 (2009). arXiv:0806.3467

33. Z. Bern, L.J. Dixon, D.A. Kosower, One loop corrections to five gluon amplitudes. Phys. Rev. Lett. 70, 2677-2680 (1993). hep-ph/9302280

34. Z. Bern, L.J. Dixon, D.C. Dunbar, D.A. Kosower, Fusing gauge theory tree amplitudes into loop amplitudes. Nucl. Phys. B 435, 59-101 (1995). hep-ph/9409265

35. G. Ossola, C.G. Papadopoulos, R. Pittau, On the rational terms of the one-loop amplitudes. JHEP 0805, 004 (2008). arXiv:0802.1876

36. M. Garzelli, I. Malamos, R. Pittau, Feynman rules for the rational part of the electroweak 1-loop amplitudes in the $R_{x} i$ gauge and in the unitary gauge. JHEP 1101, 029 (2011). arXiv:1009.4302

37. R. Pittau, Primary Feynman rules to calculate the epsilondimensional integrand of any 1-loop amplitude. JHEP 1202, 029 (2012). arXiv:1111.4965

38. K. Melnikov, M. Schulze, NLO QCD corrections to top quark pair production in association with one hard jet at hadron colliders. Nucl. Phys. B 840, 129-159 (2010). arXiv:1004.3284

39. S. Davies, One-loop QCD and higgs to partons processes using six-dimensional helicity and generalized unitarity. Phys. Rev. D 84, 094016 (2011). arXiv: 1108.0398

40. Z. Bern, D.A. Kosower, The computation of loop amplitudes in gauge theories. Nucl. Phys. B 379, 451-561 (1992)

41. Z. Bern, A. De Freitas, L.J. Dixon, H. Wong, Supersymmetric regularization, two loop QCD amplitudes and coupling shifts. Phys. Rev. D 66, 085002 (2002). hep-ph/0202271

42. C. Cheung, D. O'Connell, Amplitudes and spinor-helicity in six dimensions. JHEP 0907, 075 (2009). arXiv:0902.0981
43. F. Cascioli, P. Maierhofer, S. Pozzorini, Scattering amplitudes with open loops. arXiv:1111.5206

44. V. Hirschi, R. Frederix, S. Frixione, M.V. Garzelli, F. Maltoni et al., Automation of one-loop QCD corrections. JHEP 1105, 044 (2011). arXiv: 1103.0621

45. S. Actis, A. Denner, L. Hofer, A. Scharf, S. Uccirati, Recursive generation of one-loop amplitudes in the standard model. JHEP 1304, 037 (2013). arXiv:1211.6316

46. H.D. Vinod, Hands-On Matrix Algebra Using R. Active and Motivated Learning with Applications (World Scientific, Hackensack, 2011)

47. R.K. Ellis, W.J. Stirling, B.R. Webber, $Q C D$ and Collider Physics (Cambridge University Press, Cambridge, 1996)

48. T. Hahn, Generating Feynman diagrams and amplitudes with FeynArts 3. Comput. Phys. Commun. 140, 418-431 (2001). hep-ph/0012260

49. T. Hahn, M. Perez-Victoria, Automatized one loop calculations in four-dimensions and D-dimensions. Comput. Phys. Commun. 118 153-165 (1999). hep-ph/9807565

50. S. Agrawal, T. Hahn, E. Mirabella, FormCalc 7. J. Phys. Conf. Ser. 368, 012054 (2012). arXiv:1112.0124

51. B. Chokoufe Nejad, T. Hahn, J.N. Lang, E. Mirabella, FormCalc 8: better algebra and vectorization. arXiv: 1310.0274

52. J.A.M. Vermaseren, New features of FORM. math-ph/0010025

53. Y. Katayama, K. Sawada, S. Takagi, Five dimensional approach to regularized quantum electrodynamics. Prog. Theor. Phys. 5(1), 14-24 (1950)

54. G. 't Hooft, Renormalization of massless Yang-Mills fields. Nucl. Phys. B 33, 173-199 (1971)

55. D. Leiter, G. Szamosi, Pseudoscalar mass and its relationship to conventional scalar mass in the relativistic dirac theory of the electron. Lettere al Nuovo Cimento 5(12), 814-816 (1972)

56. M. Trzetrzelewski, On the mass term of the Dirac equation. arXiv: 1101.3899

57. U. Jentschura, B. Wundt, From generalized Dirac equations to a candidate for dark energy. ISRN High Energy Phys. 2013, 374612 (2013). arXiv:1205.0521

58. G. Mahlon, S.J. Parke, Deconstructing angular correlations in Z $\mathrm{H}, \mathrm{Z}$ Z, and W W production at LEP-2. Phys. Rev. D 58, 054015 (1998). hep-ph/9803410

59. A. Brandhuber, S. McNamara, B.J. Spence, G. Travaglini, Loop amplitudes in pure Yang-Mills from generalised unitarity. JHEP 0510, 011 (2005). hep-th/0506068

60. Z. Kunszt, A. Signer, Z. Trocsanyi, One loop helicity amplitudes for all $2 \rightarrow 2$ processes in QCD and $N=1$ supersymmetric Yang-Mills theory. Nucl. Phys. B 411, 397-442 (1994). hep-ph/9305239

61. Z. Bern, G. Chalmers, L.J. Dixon, D.A. Kosower, One loop $N$ gluon amplitudes with maximal helicity violation via collinear limits. Phys. Rev. Lett. 72, 2134-2137 (1994). hep-ph/9312333

62. Z. Bern, L.J. Dixon, D.A. Kosower, One loop corrections to two quark three gluon amplitudes. Nucl. Phys. B 437, 259-304 (1995). hep-ph/9409393

63. C.R. Schmidt, $H \rightarrow g g g(g q \bar{q})$ at two loops in the large $m_{t}$ limit. Phys. Lett. B 413, 391-395 (1997). hep-ph/9707448

64. A. van Hameren, Multi-gluon one-loop amplitudes using tensor integrals. JHEP 0907, 088 (2009). arXiv:0905.1005

65. G. Heinrich, G. Ossola, T. Reiter, F. Tramontano, Tensorial reconstruction at the integrand level. JHEP 1010, 105 (2010). arXiv: 1008.2441

66. P. Mastrolia, G. Ossola, T. Reiter, F. Tramontano, Scattering amplitudes from unitarity-based reduction algorithm at the integrandlevel. JHEP 1008, 080 (2010). arXiv:1006.0710

67. P. Mastrolia, E. Mirabella, T. Peraro, Integrand reduction of oneloop scattering amplitudes through Laurent series expansion. JHEP 1206, 095 (2012). arXiv: 1203.0291 
68. T. Peraro, Ninja: automated integrand reduction via Laurent expansion for one-loop amplitudes. Comput. Phys. Commun 185, 27712797 (2014). arXiv:1403.1229

69. G. Cullen, N. Greiner, G. Heinrich, G. Luisoni, P. Mastrolia et al., Automated one-loop calculations with GoSam. Eur. Phys. J. C 72, 1889 (2012). arXiv:1111.2034

70. L.J. Dixon, Calculating scattering amplitudes efficiently. hep-ph/9601359

71. F. Wilczek, Decays of heavy vector mesons into Higgs particles. Phys. Rev. Lett. 39, 1304 (1977)

72. S. Dawson, Radiative corrections to Higgs boson production. Nucl. Phys. B 359, 283-300 (1991)

73. S.D. Badger, Direct extraction of one loop rational terms. JHEP 01, 049 (2009). arXiv:0806.4600

74. P. Mastrolia, On triple-cut of scattering amplitudes. Phys. Lett. B 644, 272-283 (2007). hep-th/0611091

75. D. Forde, Direct extraction of one-loop integral coefficients. Phys. Rev. D 75, 125019 (2007). arXiv:0704.1835
76. R. Britto, E. Buchbinder, F. Cachazo, B. Feng, One-loop amplitudes of gluons in SQCD. Phys. Rev. D 72, 065012 (2005). hep-ph/0503132

77. R. Britto, B. Feng, P. Mastrolia, The cut-constructible part of QCD amplitudes. Phys. Rev. D 73, 105004 (2006). hep-ph/0602178

78. P. Mastrolia, Double-cut of scattering amplitudes and Stokes' theorem. Phys. Lett. B 678, 246-249 (2009). arXiv:0905.2909

79. W.B. Kilgore, One-loop integral coefficients from generalized unitarity. arXiv:0711.5015

80. R. Britto, B. Feng, Solving for tadpole coefficients in one-loop amplitudes. Phys. Lett. B 681, 376-381 (2009). arXiv:0904.2766

81. R. Britto, E. Mirabella, Single cut integration. JHEP 1101, 135 (2011). arXiv:1011.2344

82. S. Badger, E.N. Glover, V. Khoze, P. Svrcek, Recursion relations for gauge theory amplitudes with massive particles. JHEP 0507, 025 (2005). hep-th/0504159 